\title{
ABSTRACT THEORY OF ABELIAN OPERATOR ALGEBRAS: AN APPLICATION OF FORCING
}

BY

THOMAS J. JECH ${ }^{1}$

ABSTRaCT. The abstract abelian operator theory is developed from a general standpoint, using the method of forcing and Boolean-valued models.

1. Introduction. One aspect of the study of operator algebras is the description of the algebraic structure of algebras of operators, and representation of abstract algebras on a Hilbert space. This "algebraization" of the theory of algebras of operators is well understood in the case of bounded normal operators.

The theory of von Neumann algebras (or the more general $C^{*}$-algebras) is based on Stone's characterization of abelian (commutative) algebras of bounded operators in [13]. Stone's theory describes such algebras axiomatically, in algebraic terms, without reference to Hilbert space, and develops a function calculus and the spectral theory for the abstract algebras. Moreover, the algebras are algebraically isomorphic to the algebra $C(X)$ of all (complex-valued) continuous functions on an extremally disconnected compact Hausdorff space $X$.

The functional representation of abelian von Neumann algebras has been used to extend Stone's work to abelian algebras of unbounded normal operators. This has been done for instance by Fell and Kelley in [2]; a detailed account of spectral theory based on such an approach is presented by Kadison and Ringrose in [5]. For a given abelian von Neumann algebra $\mathscr{A}$, one defines an algebra $\overline{\mathscr{A}}$ of normal (not necessarily bounded) operators affiliated with $\mathscr{A}$. If $C(X)$ is the functional algebra isomorphic to $\mathscr{A}$, then $\overline{\mathscr{A}}$ is isomorphic to the algebra of all normal functions on $X$. This provides both the spectral theory and a Borel function calculus for unbounded normal operators.

Our approach is more abstract, and more in the spirit of Stone's program. We introduce an algebraic structure that we call a Stonean algebra. A Stonean algebra is essentially a normed commutative algebra with involution, but the norm does not necessarily take only finite values. Its real (i.e. selfadjoint) elements admit a partial ordering that defines a topology, generally weaker (coarser) than the topology induced by the norm. A complete Stonean algebra is complete in this topology.

We define a completion of a Stonean algebra and prove

THEOREM A. Every Stonean algebra has a unique completion.

Received by the editors February 21, 1984.

1980 Mathematics Subject Classification. Primary 03E40, 03C60, 46J99.

${ }^{1}$ Supported in part by an NSF grant. 
With the topology induced by the partial order we associate a different kind of convergence, called $\mathscr{B}$-convergence (the cognoscenti will notice that $\mathscr{B}$-convergence is the convergence in a Boolean-valued model). As $\mathscr{B}$-convergence is defined in algebraic terms, it is respected by isomorphisms between complete Stonean algebras.

As the bounded elements of a complete Stonean algebra form a complete metric space in the norm topology, this approach is a generalization of Stone's theory:

THEOREM B. Every complete Stonean algebra is isomorphic to the algebra of all normal functions on some extremally disconnected compact Hausdorff space $\mathscr{X}$; bounded elements correspond to (finite) continuous functions on $\mathscr{X}$.

We give another characterization of complete Stonean algebras, which describes the logical structure of Stonean algebras and which accounts for the formal similarity between normal operators on one hand and complex numbers on the other.

TheOREM C. Every complete Stonean algebra $\mathscr{A}$ is isomorphic to the algebra of all $\mathscr{B}$-valued complex numbers, where $\mathscr{B}$ is the complete Boolean algebra of all projections in $\mathscr{A}$.

Using this representation, one gets the following "function calculus" for complete Stonean algebras:

THEOREM D. For any element a of a complete Stonean algebra $\mathscr{A}$ there is a homomorphism $\varphi_{a}$ of the algebra of all Borel (complex-valued) functions on $\mathbf{C}$ into $\mathscr{A}$ such that

(a) $\varphi_{a}$ (id) $=a$, and

(b) if $f$ is a pointwise limit of $\left\{f_{n}\right\}$, then $\varphi_{a}(f)$ is the $\mathscr{B}$-limit of $\left\{\varphi_{a}\left(f_{n}\right)\right\}$.

This justifies the customary notation

$$
f(a)=\varphi_{a}(f) \quad(a \in \mathscr{A}, f \text { Borel on } \mathbf{C}) .
$$

We shall now turn our attention to operator algebras. The following theorem is the raison d'être for the concept of a complete Stonean algebra.

THEOREM E. For any set $G$ of mutually compatible normal operators in a Hilbert space, there exists a smallest complete Stonean algebra $\mathscr{A}$ of normal operators such that $G \subseteq \mathscr{A}$.

The construction of $\mathscr{A}$ in Theorem $\mathrm{E}$ is well known; cf. [8]. If $G$ consists of a single unbounded normal operator, $\mathscr{A}$ is the algebra constructed in [2]; if $G$ is an abelian von Neumann algebra, $\mathscr{A}$ is the algebra constructed in [5].

Not every complete Stonean algebra is isomorphic to a complete algebra of operators. A necessary condition is that its Boolean algebra of projections is the direct sum of measure algebras. We call a Stonean algebra measurable if its $B$ is a measure algebra. 
THEOREM F. Every measurable complete Stonean algebra is isomorphic to the algebra of all measurable functions $(\bmod =$ a.e. $)$ on some measure space $\mathscr{X}$; bounded elements correspond to functions in $\mathscr{L}_{\infty}(\mathscr{X})$.

This generalizes the well-known representation of abelian von Neumann algebras on a separable Hilbert space by $\mathscr{L}_{\infty}$; cf. [14, Theorem 1.22].

Let $\mathscr{A}$ be a measurable complete Stonean algebra and let $\mathscr{H}$ be a Hilbert space. We say that $\mathscr{A}$ has a complete representation on $\mathscr{H}$ if there is a complete abelian algebra $\mathscr{A}^{\prime}$ of operators in $\mathscr{H}$ such that $\mathscr{A}$ and $\mathscr{A}^{\prime}$ are isomorphic (as complete Stonean algebras).

THEOREM G. Every measurable complete Stonean algebra $\mathscr{A}$ has a complete representation on some Hilbert space $\mathscr{H}$; if $\mathscr{A}$ is countably generated, then there is such an $\mathscr{H}$ that is separable.

Some of the theory presented in this paper owes much to the theory of Booleanvalued models, which, of course, descends from Cohen's method of forcing [1]. I am particularly indebted to Scott and Solovay's treatment of real numbers in Booleanvalued models [11], as well as to Takeuti's application of these methods to selfadjoint operators [15]. I am also grateful to Richard Herman for many enlightening discussions on operator algebras.

In $\$ 2$ we state axioms for Stonean algebras and complete Stonean algebras, and show that a complete Stonean algebra $\mathscr{A}$ is determined by the complete Boolean algebra of projections in $\mathscr{A}$.

In $\S 3$ we introduce the norm and piecewise uniform and $\mathscr{B}$-convergence in complete Stonean algebras.

In $\$ 4$ we introduce the Boolean-valued model and show that the $\mathscr{B}$-valued complex numbers form a complete Stonean algebra with projections $\mathscr{B}$.

In $\$ 5$ (representation by continuous functions) we prove Theorem B and investigate the two kinds of convergence on the function algebra.

$\S 6$ introduces the function calculus.

$\$ 7$ is devoted to the construction of a completion of a Stonean algebra.

In $\S 8$ we generalize the definition of $\mathscr{B}$-convergence for Stonean algebras that are not necessarily complete, and prove that completeness is equivalent to $\mathscr{B}$-convergence of all $\mathscr{B}$-Cauchy nets. We also describe the complete Stonean subalgebra of a complete Stonean algebra generated by a given set.

In $\$ 9$ we introduce measurable Stonean algebras, prove Theorem F, show that $\mathscr{B}$-convergence for measurable algebras corresponds to convergence of functions a.e., and $\mathscr{B}$-convergence and piecewise uniform convergence coincide.

In $\$ 10$ we apply the general theory to complete abelian algebras of operators in a Hilbert space.

2. Complete Stonean algebras. We introduce axioms for Stonean algebras and complete Stonean algebras. Throughout the paper, we use lower case Greek letters for complex numbers (scalars). Several of the axioms are redundant when completeness is assumed. 
Let $\mathscr{A}$ be a set containing 0 and 1 , endowed with addition, multiplication, scalar multiplication and involution ${ }^{*}$, and a binary relation $\leqslant$.

2.1 Axiom I. $\mathscr{A}$ is an abelian algebra over $\mathrm{C}$ with involution $*$ and unit 1.

For every $\lambda \in \mathbf{C}$ we identify $\lambda$ with the element $\lambda \cdot 1 \in \mathscr{A}$; thus + and $\cdot$ agree with complex + and $\cdot$, and $\lambda^{*}=\bar{\lambda}$.

2.2 Definition. An element $a \in \mathscr{A}$ is real if $a^{*}=a$.

We denote the set of all reals by $\mathscr{R}$. Every $z \in \mathscr{A}$ has the real part $x=\operatorname{Re}(z)=$ $\left(z+z^{*}\right) / 2$, and the imaginary part $y=\operatorname{Im}(z)=\left(z-z^{*}\right) / 2 i$, where $x, y \in \mathscr{R}$ and $z=x+i y$. For every $z=x+i y$, the element $z z^{*}=x^{2}+y^{2}$ is real.

2.2 AxIOM II. The relation $\leqslant$ is a partial ordering of $\mathscr{R}$ and satisfies:

(a) if $a \leqslant b$, then $a+c \leqslant b+c(a, b, c \in \mathscr{R})$,

(b) if $a \geqslant 0$ and $b \geqslant 0$, then $a \cdot b \geqslant 0(a, b \in \mathscr{R})$,

(c) if $\lambda \geqslant 0$, then $\lambda \geqslant 0$,

(d) $a^{2} \geqslant 0$ for all $a \in \mathscr{R}, a^{2}=0$ only if $a=0$,

(e) if $a, b \in \mathscr{R}$, then $\{a, b\}$ has a least upper bound $a \vee b$,

(f) if $u \geqslant 0$ and $a, b \in \mathscr{R}$, then $u \cdot(a \vee b)=(u a) \vee(u b)$,

(g) if $u>0$, then $u \wedge 1>0$.

Thus $\leqslant$ agrees on $\mathbf{R}$ with the natural ordering of real numbers. Note that, by 2.2(d), $z z^{*} \geqslant 0$ for all $z \in \mathscr{A}$.

As usual, $a>b$ means $a \geqslant b$ and $a \neq b$. We call a real $u$ positive if $u>0$.

2.3 Ахіом III. Let a be real. If $a \nless 0$ then there is a positive $u$ such that $u a>0$.

The following axiom guarantees that $\mathscr{A}$ has no infinitesimals.

2.4 Aхіом IV. Let a be real. If $a \leqslant \varepsilon$ for all $\varepsilon>0$, then $a \leqslant 0$.

2.5 Ахіом V. Let a be real. For every positive real $u$ there is a positive $v \leqslant u$ and $\lambda \in \mathbf{R}$ such that av $\leqslant \lambda$.

(Every element of $\mathscr{A}$ is locally bounded.)

We call $\mathscr{A}$ a Stonean algebra if it satisfies Axioms I-V. The following two axioms make a Stonean algebra complete.

2.6 Axiom VI. Let $A$ be a nonempty set of nonnegative reals such that $a \cdot b=0$ whenever $a, b$ are distinct elements of $A$. Then there is some $u \in \mathscr{R}$ such that $u \geqslant a$ for all $a \in A$.

Such a $u$ is an upper bound of the set $A$ in the partial ordering $\leqslant$.

2.7 AхIOM VII. Every nonempty set of reals that has an upper bound in $\leqslant$ has a least upper bound. If $u \geqslant 0$, then $\sup \{u a: a \in A\}=u \cdot \sup A$.

We refer to Axioms VI and VII as the completeness axioms.

2.8 Definition. A homomorphism of a Stonean algebra preserves $+, \cdot,{ }^{*}$ and $\leqslant$. A complete homomorphism preserves least upper bounds whenever they exist. A subalgebra is a regular subalgebra if the inclusion homomorphism is complete. 
2.9 EXAMPLES. (a) $\mathbf{C}$ is a complete Stonean algebra and is a regular subalgebra of every Stonean algebra.

(b) $\mathscr{L}_{\infty}(0,1)$ is a Stonean algebra, not complete.

(c) $\mathscr{M}(0,1)$, the algebra of all measurable functions on $(0,1)$, is a complete Stonean algebra, and $\mathscr{L}_{\infty}(0,1)$ is a regular subalgebra of $\mathscr{M}(0,1)$.

(d) $\mathrm{C}[0,1]$ is a Stonean algebra and a subalgebra of $\mathscr{L}_{\infty}(0,1)$ but not an regular subalgebra.

The claim in 2.9(a) is just a restatement of Axiom IV:

2.10 Lemma. For all $\lambda \in \mathbf{R}, \lambda=\sup \{\mu: \mu<\lambda\}$.

Proof. By Axiom IV, $0=\inf \{\varepsilon: \varepsilon>0\}$.

2.11 Definition. A projection $p$ is real such that $p^{2}=p$. We denote by $\mathscr{B}$ the set of all projections.

2.12 Lemma. The set $\mathscr{B}$ is a Boolean algebra; if $\mathscr{A}$ is a complete Stonean algebra, then $\mathscr{B}$ is a complete Boolean algebra.

Proof. The Boolean algebraic operations on $\mathscr{B}$ are defined as follows:

$$
p \wedge q=p \cdot q, \quad p \vee q=p+q-p \cdot q, \quad-p=1-p .
$$

An easy calculation shows that these operations, applied to projections, yield projections. Likewise, the standard axioms of Boolean algebras [9] are verified. For instance, the distributive and de Morgan laws read

$$
\begin{aligned}
& p(q+r-q \cdot r)=p q+p r-(p q)(p r), \\
& 1-p q=(1-p)+(1-q)-(1-p)(1-q),
\end{aligned}
$$

and the partial ordering $\leqslant$ of $\mathscr{R}$ is the Boolean algebraic partial ordering of $\mathscr{B}$ :

$$
p \leqslant q \text { iff } p \cdot q=p .
$$

To verify (2.15), note first that by $2.2(\mathrm{~d}), p \geqslant 0$ for all $p \in \mathscr{B}$, and hence $0 \leqslant p \leqslant 1$. If $p \cdot q=p$, then $p=p q \leqslant 1 \cdot q=q$; conversely, if $p \leqslant q$, then $p=p^{2} \leqslant p q \leqslant p \cdot 1$ $=p$, and so $p q=p$.

If $S$ is a nonempty set of projections, then 1 is an upper bound; if $p=\sup S$, then $p$ is a projection.

The main result of this section is that a complete Stonean algebra is uniquely determined by its Boolean algebra of projections.

2.16 Remark. In the Examples $2.9, \mathscr{B}=\{0,1\}$ when $\mathscr{A}=\mathbf{C}$ or $\mathscr{A}=\mathbf{C}[0,1]$, and $\mathscr{B}=$ Borel $/$ null when $\mathscr{A}=\mathscr{L}_{\infty}$ or $\mathscr{M}$.

2.17 ThEOREM. Let $\mathscr{A}_{1}$ and $\mathscr{A}_{2}$ be complete Stonean algebras and let $\mathscr{B}_{1}$ and $\mathscr{B}_{2}$ be their algebras of projections. If $\pi$ is an isomorphism between $\mathscr{B}_{1}$ and $\mathscr{B}_{2}$, then $\pi$ has a unique extension to an isomorphism between $\mathscr{A}_{1}$ and $\mathscr{A}_{2}$.

We devote the rest of the section to the proof of Theorem 2.17; we assume that $\mathscr{A}$ is a complete Stonean algebra.

2.18 Definition. A condition is a projection $p \neq 0$. A condition $p$ is stronger than a condition $q$ if $p \leqslant q$. The set of all conditions is denoted by $P$. 
2.19 Definition. Let $a, b$ be reals and $p, q$ conditions:

$$
\begin{array}{ll}
p \Vdash a=b(p \text { forces } a=b) & \text { if } p a=p b, \\
p \Vdash a \leqslant b & \text { if } p a \leqslant p b, \\
p \Vdash a \neq b & \text { if } q a \neq q b \text { for every } q \text { stronger than } p, \\
p \Vdash a<b & \text { if } p \Vdash a \leqslant b \text { and } p \Vdash a \neq b .
\end{array}
$$

(Of course, the inspiration for this definition is Cohen's forcing [1].)

2.20 LEMMA. (a) If $p$ forces $a=b(a \leqslant b, a \neq b, a<b)$ and if $q$ is stronger than $p$, then $q$ forces $a=b(a \leqslant b, a \neq b, a<b)$.

(b) No p forces both $a=b$ and $a \neq b$.

(c) $a=b$ iff $1 \Vdash a=b$ iff every $p \Vdash a=b ; a \leqslant b$ iff $1 \Vdash a \leqslant b$ iff every $p \Vdash a \leqslant b$.

Proof. Easy.

2.21 Definition.

$$
\begin{array}{ll}
\llbracket a=b \rrbracket=\sup \{p: p \Vdash a=b\}, & \llbracket a \leqslant b \rrbracket=\sup \{p: p \Vdash a \leqslant b\}, \\
\llbracket a \neq b \rrbracket=\sup \{p: p \Vdash a \neq b\}, & \llbracket a<b \rrbracket=\sup \{p: p \Vdash a<b\} .
\end{array}
$$

2.22 Lemma. For every $a, b$ and $p$, there is $a q \leqslant p$ such that $q$ forces $a=b$, or $q$ forces $a \neq b$.

COROLlaRy. $\llbracket a \neq b \rrbracket=1-\llbracket a=b \rrbracket$.

Proof. If there is no condition $q \leqslant p$ that forces $a=b$, then $p$ forces $a=b$ by definition.

2.23 Lemma. For every $a, b$ and $p$, there is $a q \leqslant p$ such that $q$ forces $a \leqslant b$ or $q$ forces $b \leqslant a$.

COROLlaRY. $\llbracket a<b \rrbracket=1-\llbracket b \leqslant a \rrbracket$.

Proof. Without loss of generality, assume that $b=0$. Let $p \in P$. By Axiom III there exists a positive $v \leqslant p$ such that either $a v \geqslant 0$ or $a v \leqslant 0$. Let us assume that $a v \geqslant 0$. Let $q=\llbracket v \neq 0 \rrbracket$. As $v \neq 0, q$ is a condition; we shall show that $q \leqslant p$ and $q \Vdash a \geqslant 0$. If $r$ is a condition such that $r \cdot p=0$, then $r v \leqslant r p=0$, and so $r \Vdash v=0$; hence $r \cdot q=0$ and it follows that $q \leqslant p$.

As $1-q=\llbracket v=0 \rrbracket$, we have $(1-q) \cdot v=0$, and so $v \leqslant q$. Consequently, $q a \geqslant$ $v a \geqslant 0$, and $q \Vdash a \geqslant 0$.

2.24 LemMA. If $a>0$, then there is a condition $p$ and some $\varepsilon>0$ such that $p \Vdash a \geqslant \varepsilon$.

Proof. By Axiom IV there is an $\varepsilon>0$ such that $a \nless \varepsilon$. By Lemma 2.23 it follows that $\llbracket a \leqslant \varepsilon \rrbracket \neq 1$, and so there is a $p \in P$ that forces $a \geqslant \varepsilon$.

2.25 LEMMA. For every real $a$ and every condition $p$, there is a stronger condition $q$ and some $\lambda \in \mathbf{R}$ such that $q \Vdash a \leqslant \lambda$.

Proof. Let $p \in P$. By Axiom $\mathrm{V}$ there exists a positive $v \leqslant p$ and some $\mu$ such that $a v \leqslant \mu$. By Lemma 2.24 there is a $q$ and an $\varepsilon>0$ such that $q \Vdash v \geqslant \varepsilon$. Because 
$v \leqslant p$ we have (as in the Proof of Lemma 2.23) $q \leqslant p ; \varepsilon q a \leqslant q a v \leqslant \mu q$, hence $q \Vdash \varepsilon a \leqslant \mu$, and letting $\lambda=\mu / \varepsilon$ we get $q \Vdash a \leqslant \lambda$.

2.26 Definition. Two projections $p$ and $q$ are orthogonal if $p \cdot q=0$. A partition of unity is a maximal set of pairwise orthogonal conditions (nonzero projections).

2.27 Lemma. Let $W$ be a partition of unity and let $\left\{a_{p}: p \in W\right\} \subset \mathscr{A}$. Then there is a unique $a \in \mathscr{A}$ such that $p a=p a_{p}$ for all $p \in W$.

Proof. It is enough to prove the existence in the case when each $a_{p} \geqslant 0$; this is because every element of $\mathscr{A}$ has the form $x+i y$ where $x, y \in \mathscr{R}$, and every real has the form $a=a^{+}+a^{-}$where $a^{+}=a \cdot \llbracket a \geqslant 0 \rrbracket \geqslant 0$ and $a^{-}=a \cdot \llbracket a \leqslant 0 \rrbracket \leqslant 0$.

Thus let $W$ be a partition of unity, and $a_{p} \geqslant 0$ for all $p \in W$. By Axiom VI, the set

$$
A=\left\{p a_{q}: p \in W\right\}
$$

has an upper bound, and hence a least upper bound $a=\sup A$. It is easily verified that $p a=p a_{p}$ for all $p \in W$.

To prove uniqueness, it suffices to show that $a=0$ when $p a=0$ for all $p \in W$. But then $0=\sup \{p a: p \in W\}=a \cdot \sup W=a \cdot 1=a$.

2.28 Definition. A step function is an element $s$ with the property that there is a partition of unity $W$ and a set $\left\{\lambda_{p} \in \mathbf{C}: p \in W\right\}$ such that $s p=\lambda_{p} p$ for all $p \in W$.

2.29 Definition. Let $\mathscr{A}_{1}, \mathscr{A}_{2}$ be Stonean algebras and let $\mathscr{A}_{1}$ be a subalgebra of $\mathscr{A}_{2} . \mathscr{A}_{2}$ is dense in $\mathscr{A}_{2}$ if:

(a) for every $a_{2}>0$ in $\mathscr{A}_{2}$ there is an $a_{1}>0$ in $\mathscr{A}_{1}$ such that $a_{1} \leqslant a_{2}$, and

(b) for every real $a_{2}$ in $\mathscr{A}_{2}$ there is an $a_{1} \in \mathscr{A}_{1}$ such that $a_{1} \geqslant a_{2}$.

2.30 Lemma. If $\mathscr{A}_{1}$ is a dense subalgebra of a complete Stonean algebra $\mathscr{A}_{2}$, then every real $a \in \mathscr{A}_{2}$ is the least upper bound of the set

$$
S=\left\{x \in \mathscr{A}_{1}: x \leqslant a\right\} .
$$

Corollary. If $\mathscr{A}_{1}$ is a dense subalgebra of a complete Stonean algebra $\mathscr{A}_{2}$, then $\mathscr{A}_{1}$ is a regular subalgebra of $\mathscr{A}_{2}$.

Proof. Let $a \in \mathscr{A}_{2}$. By 2.29(b) there is an $x \in \mathscr{A}_{1}$ such that $x \leqslant a$, and so the set $S$ is nonempty. Let $b=\sup S$; we have $b \leqslant a$. If $b<a$, let $d>0$ be in $\mathscr{A}_{2}$ such that $d \leqslant a-b$. Then $x+d \in S$ for all $x \in S$, and

$$
b+d=\sup \{x+d: x \in S\} \leqslant b,
$$

a contradiction.

2.31 Lemma. Let $\mathscr{A}$ be a complete Stonean algebra. The set of all step functions in $\mathscr{A}$ is a dense subalgebra of $\mathscr{A}$.

Proof. Lemmas 2.24, 2.25 and 2.27.

2.32. We are now ready to complete the proof of Theorem 2.17 . Let $\mathscr{A}_{1}$ and $\mathscr{A}_{2}$ be complete Stonean algebras and let us assume that $\mathscr{A}_{1}$ and $\mathscr{A}_{2}$ have the same algebra 
$\mathscr{B}$ of projections. We extend the identity mapping on $\mathscr{B}$ to an isomorphism $\pi$ between $\mathscr{A}_{1}$ and $\mathscr{A}_{2}$ as follows:

We let $\pi(s)=s$ for every step function $s \in \mathscr{A}_{1}$. Then we apply Lemma 2.31 to extend $\pi$ to an isomorphism between $\mathscr{A}_{1}$ and $\mathscr{A}_{2}$. This also proves uniqueness because if $\pi$ is an isomorphism between $\mathscr{A}_{1}$ and $\mathscr{A}_{2}$, then: (a) $\pi(1)=1$, (b) $\pi(n)=n$ for all $n=1,2,3, \ldots$, (c) $\pi(r)=r$ for every rational $r$, (d) $\pi(\lambda)=\lambda$ for every $\lambda \in \mathbf{R}$, and, finally, (e) $\pi(s)=s$ for every step function $s$.

3. Norm and convergence. Throughout this section, $\mathscr{A}$ is a complete Stonean algebra. For each $a \in \mathscr{A}$ we define the norm of $a$ as follows:

$$
\|a\|=\left\{\begin{array}{l}
\text { least } \lambda \geqslant 0 \text { such that } a a^{*} \leqslant \lambda^{2} \text { if such a } \lambda \text { exists, } \\
\infty \text { otherwise. }
\end{array}\right.
$$

3.2 LemMA. (a) $\|a\| \geqslant 0$, and $\|a\|=0$ iff $a=0$,

(b) $\|\lambda a\|=|\lambda| \cdot\|a\|$,

(c) $\|a+b\| \leqslant\|a\|+\|b\|$,

(d) $\|a \cdot b\| \leqslant\|a\| \cdot\|b\|$, and

(e) $\left\|a a^{*}\right\|=\|a\|^{2}$.

Proof. Follows from Axiom II(d). (b) and (d) are easy, and (c) is a standard algebraic calculation. To prove (e), we can reduce it to the case when $a>0$ and $\left\|a^{2}\right\|=1$; we want to prove that $a \leqslant 1$. If not, then there is a $p \in P$ and an $\varepsilon>0$ such that $p \Vdash a \geqslant 1+\varepsilon$, and so $p \Vdash a^{2} \geqslant(1+\varepsilon)^{2}$, which implies $\left\|a^{2}\right\|>1$.

3.3 Definition. The set $B(\mathscr{A})$ of all bounded elements of $\mathscr{A}$ consists of all $a \in$ $\mathscr{A}$ of finite norm. $B(\mathscr{A})$ is the bounded part of $\mathscr{A}$.

The bounded part of $\mathscr{A}$ is a regular subalgebra of $\mathscr{A}$. The norm induces a metric on $B(\mathscr{A})$ which, due to the completeness axiom (Axiom VII) makes $B(\mathscr{A})$ a complete metric space, and therefore a $C^{*}$-algebra [14].

We shall consider three kinds of convergence of sequences in $\mathscr{A}$, and, more generally, of nets in $\mathscr{A}$ (see [6] for definition of a net). [In $\S 8$ we give a general definition for algebras that are not necessarily complete.]

3.4 Definition. (a) $a$ is a uniform limit of $\left\{a_{n}\right\}$ if $\left\|a_{n}-a\right\| \rightarrow 0$,

(b) $a$ is a piecewise uniform limit of $\left\{a_{n}\right\}$ if there is a partition of unity $W$ such that, for all $p \in W,\left\{a_{n} p\right\}$ converges uniformly to $a p$, and

(c) $a$ is a $\mathscr{B}$-limit of $\left\{a_{n}\right\}$ if for every $\varepsilon>0$ there is a partition of unity $W_{\varepsilon}$ and a set $\left\{N_{p}^{\varepsilon}: p \in W_{\varepsilon}\right\}$ such that, for every $p \in W_{\varepsilon}, n \geqslant N_{p}^{\varepsilon}$ implies $\left\|a p-a_{n} p\right\| \leqslant \varepsilon$.

An obvious modification of 3.4 yields the definition of uniformly Cauchy, piecewise uniformly Cauchy, and $\mathscr{B}$-Cauchy sequences and nets.

A more or less standard argument shows that a limit (of each kind), if it exists, is unique. The completeness axiom yields the expected:

3.5 LeMMA. (a) Every uniformly Cauchy net has a uniform limit.

(b) Every piecewise uniformly Cauchy net has a piecewise uniform limit.

(c) Every $\mathscr{B}$-Cauchy net has a $\mathscr{B}$-limit. 
Proof. By treating the real and imaginary parts separately, we may assume that all the elements involved are real.

(a) If $\left\{a_{n}\right\}$ is uniformly Cauchy, then the set $\left\{a_{n}\right\}$ has a lower bound and an upper bound. It is easily verified that $a=\inf _{m} \sup _{n \geqslant m}\left\{a_{m}\right\}$ is a uniform limit of the net $\left\{a_{n}\right\}$.

(b) This is a piecewise version of (a).

(c) Let $\left\{a_{n}\right\}$ be a $\mathscr{B}$-Cauchy. There is a partition of unity $W$ and, for all $p \in W$, there is an $N_{p}$ such that $\left\|a_{n} p-a_{m} p\right\| \leqslant 1$ whenever $n, m \geqslant N_{p}$. Let

$$
b_{n} p=\left\{\begin{array}{ll}
a_{n} p & \text { if } n \geqslant N_{p}, \\
a_{N_{p}} p & \text { otherwise }
\end{array} \quad(p \in W)\right.
$$

and also

$$
a p=a_{N_{p}} p \quad(p \in W) .
$$

The set $\left\{b_{n}\right\}$ has a lower bound $a-1$, an upper bound $a+1$, and a $\mathscr{B}$-limit $b=\inf _{n} \sup _{n \geqslant m}\left\{b_{n m}\right\}$. And $b$ is also the $\mathscr{B}$-limit of $\left\{a_{n}\right\}$.

It is clear that uniform convergence implies piecewise uniform convergence which, in turn, implies $\mathscr{B}$-convergence.

EXAMPLE. Let $a>0,\|a\|=\infty$, and let $a_{n}=1 / n \cdot a \cdot \llbracket a \leqslant n^{2} \rrbracket$. then $a_{n} \rightarrow 0$ piecewise uniformly, but $\left\|a_{n}\right\|=n \rightarrow \infty$.

We shall show later that, for operator algebras, $\mathscr{B}$-convergence and piecewise uniform convergence coincide.

We conclude with the following observation.

3.6 Proposition. Let $\mathscr{A}$ be a complete Stonean algebra. Every $a \in \mathscr{A}$ is a piecewise uniform limit of a sequence in $B(\mathscr{A})$.

Proof. Assume that $a \geqslant 0$. For every $n$, let $a_{n}=a \cdot \llbracket a \leqslant n \rrbracket=a \wedge n$. The general case is reduced to $a \geqslant 0$ by using $\operatorname{Re}(z)$, and $a \cdot \llbracket a \geqslant 0 \rrbracket+a \cdot \llbracket a \leqslant 0 \rrbracket$.

4. The Boolean-valued model $V^{\mathscr{B}}$. We proved in Theorem 2 that a complete Stonean algebra is determined by its algebra of projections. We shall now give an explicit construction of a complete Stonean algebra from its projections. The technique is known: in the context of operators on Hilbert space it is the spectral resolution $\int \lambda d p_{\lambda}$ (see e.g. [8]); in set theory it is the construction of a Booleanvalued model (see e.g. [4]).

4.1. Let $\mathscr{B}$ be a complete Boolean algebra. To construct a complete Stonean algebra $\mathscr{A}=\mathscr{A}(\mathscr{B})$ whose projections are isomorphic to $\mathscr{B}$, we define its reals $\mathscr{R}(\mathscr{A})=\mathscr{R}(\mathscr{B})$. An element of $\mathscr{R}(\mathscr{B})$ is a function $\left\langle p_{\lambda}: \lambda \in \mathbf{R}\right\rangle$ with values in $\mathscr{B}$ such that:

$$
\begin{aligned}
& \text { (i) } p_{\lambda} \leqslant p_{\mu} \text { if } \lambda \leqslant \mu, \\
& \text { (ii) } \bigwedge_{\lambda \in \mathbf{R}} p_{\lambda}=0, \bigvee_{\lambda \in \mathbf{R}} p_{\lambda}=1, \text { and } \\
& \text { (iii) } p_{\lambda}=\bigwedge_{\varepsilon>0} p_{\lambda+\varepsilon} \text {. }
\end{aligned}
$$


Our intention is, of course, that a function $\left\langle p_{\lambda}: \lambda \in \mathbf{R}\right\rangle$ represents the (unique) real in a complete Stonean algebra $\mathscr{A}$ such that

$$
\|a \leqslant \lambda\|=p_{\lambda} \quad(\lambda \in \mathbf{R}) .
$$

If $a \in \mathscr{A}$, then the function defined by (4.3) has properties (4.2); conversely, if $\left\langle p_{\lambda}\right\rangle$ has properties (4.2), then there is a unique $a \in \mathscr{A}$ such that (4.3) holds, namely

$$
a=\sup \left\{\lambda p_{\lambda}: \lambda \in \mathbf{R}\right\} \text {. }
$$

Thus to construct a complete Stonean algebra from $\mathscr{B}$, it suffices to endow the set $\mathscr{R}(\mathscr{B})$ with appropriate operations and,$+ \cdot$ and relation $\leqslant($ as scalar multiplication is obvious), and verify that the resulting $\mathscr{A}(\mathscr{B})$ satisfies Axioms I through VII.

We could do that by exploiting known identities for spectral resolutions (see [15] for details). We follow a different path, and use the Boolean-valued model $V^{\mathscr{B}}$ instead.

The Boolean-valued model of set theory is a class $V^{\mathscr{B}}$ of objects ( $\mathscr{B}$-valued names), endowed with $\mathscr{B}$-valued functions (of two variables)

$$
\llbracket x=y \rrbracket, \quad \llbracket x \in y \rrbracket .
$$

The actual construction of $V^{\mathscr{B}}$ is irrelevant (see [4] for details), and for our purposes it is convenient to assume that $\llbracket x=y \rrbracket=1$ holds only when $x=y$ (this can be assumed because $\llbracket x=y \rrbracket=1$ is an equivalence relation). The important property of the Boolean-valued model is this: From (4.5) one defines Boolean values of all statements expressible in the language of set theory, by

$\llbracket \Phi$ and $\Psi \rrbracket=\llbracket \Phi \rrbracket \wedge \llbracket \Psi \rrbracket, \llbracket \Phi$ or $\Psi \rrbracket=\llbracket \Phi \rrbracket \vee \llbracket \Psi \rrbracket, \llbracket \operatorname{not} \Phi \rrbracket=1-\llbracket \Phi \rrbracket$,

$\llbracket$ there is $x$ such that $\Phi(x) \rrbracket=\bigvee\left\{\llbracket \Phi(x) \rrbracket: x \in V^{\mathscr{B}}\right\}$,

$\llbracket$ for all $x$ such that $\Phi(x) \rrbracket=\bigwedge\left\{\llbracket \Phi(x) \rrbracket: x \in V^{\mathscr{B}}\right\}$.

We see that $\Phi$ holds in $V^{\mathscr{B}}$ if $\llbracket \Phi \rrbracket=1$. The main tool of the Boolean-valued model theory is that every axiom of set theory (and hence every theorem of set theory) holds in every Boolean-valued model.

The method of Boolean-valued models was developed by Scott and Solovay [11] and Vopěnka [16] from Cohen's method of forcing. In current terminology, when we let $P=\mathscr{B}-\{0\}$ ( forcing conditions),

$$
p \Vdash \Phi(p \text { forces } \Phi) \text { means } p \leqslant \llbracket \Phi \rrbracket \text {. }
$$

One technical feature of $V^{\mathscr{B}}$ is useful to mention, that is, the property

(4.8) if $\llbracket \exists x \Phi(x) \rrbracket=1$, then there is an $x \in V^{\mathscr{D}}$ such that $\llbracket \Phi(x) \rrbracket=1$.

In view of (4.6), this is a consequence of the following property:

$$
\begin{aligned}
& \text { If } W \text { is a partition of unity and }\left\{x_{p}: p \in W\right\} \subset V^{\mathscr{B}} \text {, then } \\
& \text { there is a unique } x \in V^{\mathscr{B}} \text { such that } p \Vdash x=x_{p} \text {, for all }
\end{aligned}
$$$$
p \in W \text {. }
$$

(Compare with Lemma 2.27.) It also follows from (4.9) that if $x$ is obtained from $\left\{x_{p}: p \in W\right\}$ and if $\Phi$ is some property such that $\llbracket \Phi\left(x_{p}\right) \rrbracket=1$ for all $p$, then $\llbracket \Phi(x) \rrbracket=1$. For example, the universe of sets $V$ (the ground model) is naturally embedded in $V^{\mathscr{B}}$ as a subclass $V \subset V^{\mathscr{B}}$. By (4.9) it follows that

$$
\llbracket x \in V \rrbracket=1
$$


if and only if there is a partition of unity $W$ and a set $\left\{x_{p}: p \in W\right\} \subset V$ such that $\llbracket x=x_{p} \rrbracket=p$ for all $p \in W$.

Let $\mathbf{R}^{\mathscr{B}}$ be the set of all $\mathscr{B}$-valued reals, that is, the set of all $a \in V^{\mathscr{B}}$ such that

$\llbracket a$ is a real number $=1$.

The actual real numbers $\mathbf{R}$ (the real numbers in $V$ ) are embedded in $\mathbf{R}^{\mathscr{B}}$ as a dense subset; they are those $a \in V^{\mathscr{B}}$ which satisfy

$$
\llbracket a \in \mathbf{R} \rrbracket=1,
$$

which by (4.10) is equivalent to being a "step function":

$$
\llbracket a=\lambda_{p} \rrbracket=p \quad(p \in W ; W \text { a partition of unity }) .
$$

As $\llbracket \mathbf{R}$ is dense in $\mathbf{R}^{\mathscr{B}} \rrbracket=1$, every $a \in \mathbf{R}^{\mathscr{B}}$ is uniquely determined by the set of all $\lambda \in \mathbf{R}$ with $a \leqslant \lambda$, i.e., by the $\mathscr{B}$-valued function

$$
\langle\llbracket a \leqslant \lambda \rrbracket: \lambda \in \mathbf{R}\rangle .
$$

The function (4.12) satisfies (4.2); conversely any function that satisfies (4.2) defines an element $a$ of $\mathbf{R}^{\mathscr{B}}$, and such an $a$ is unique by the assumption we made on the equivalence relation $\llbracket x=y \rrbracket=1$. Thus we have a one-to-one correspondence between $\mathbf{R}^{\mathscr{P}}$ and $\mathscr{R}$. (This correspondence is due to Scott and Solovay.)

Thus $\mathscr{R}$ is identified with $\mathbf{R}^{\mathscr{P}}$ which is naturally endowed with,$+ \cdot$ and $\leqslant$ :

$$
\begin{array}{ll}
a \leqslant b & \text { if } \llbracket a \leqslant b \rrbracket=1, \\
a+b=c & \text { if } \llbracket a+b=c \rrbracket=1, \\
a \cdot b=c & \text { if } \llbracket a \cdot b=c \rrbracket=1 .
\end{array}
$$

Let $\mathscr{A}$ be the algebra of all $a+i b$ where $a, b \in \mathscr{R}$.

4.14 Lemma. $\mathscr{A}$ is a complete Stonean algebra and its algebra of projections is $\mathscr{B}$.

Proof. Axioms I and II of $\$ 2$ are easily verified using the calculus of Boolean values (4.6).

Proof of Axiom III. Let $a \in \mathscr{R}$. The statement

holds in $V^{\mathscr{P}}$, and so

$$
a \leqslant 0 \text { or } a \geqslant 0
$$

$$
\llbracket a \leqslant 0 \rrbracket \vee \llbracket a \geqslant 0 \rrbracket=1 .
$$

If $a \nless 0$ (i.e. if $\llbracket a \leqslant 0 \rrbracket \neq 1$ ), then $\llbracket a \geqslant 0 \rrbracket>0$. Let $p=\llbracket a \geqslant 0 \rrbracket$. Then $p \cdot a=$ the unique $b$ such that $\llbracket b=a \rrbracket=p$ and $\llbracket b=0 \rrbracket=1-p$, and we have $p a \geqslant 0$ for this $p>0$.

Proof of Axiom IV. If $a<\varepsilon$ for all $\varepsilon>0$, then

$$
\llbracket a \leqslant \varepsilon \text { for all } \varepsilon \in \mathbf{R}, \varepsilon>0 \rrbracket=1 \text {. }
$$

and hence

$$
\llbracket a \leqslant 0 \rrbracket=1 .
$$

Proof of Axiom V. Let $a \in \mathscr{R}$ and let $u>0$. Since

$$
\llbracket \text { there is } \lambda \in \mathbf{R} \text { such that } a u \leqslant \lambda \rrbracket=1,
$$

there exists $\lambda \in \mathbf{R}$ and $p \in \mathscr{B}, p>0$, such that

$$
p \Vdash u>0 \text { and } a u \leqslant \lambda .
$$

Let $v=p u$. Then $0<v \leqslant u$, and $a v \leqslant \lambda$. 
Proof of Axiom VI. Let $A$ be a nonempty set of nonnegative reals such that $a \cdot b=0$ whenever $a, b \in A$. For each $a \in A$ let

$$
p_{a}=\llbracket a \neq 0 \rrbracket .
$$

Since

$$
\llbracket a \cdot b=0 \text { implies either } a=0 \text { or } b=0 \rrbracket=1,
$$

it follows that $p_{a} \cdot p_{b}=0$ whenever $a \neq b$. Let $p_{0}=1-\bigvee_{a \in A} p_{a}$ and let $W=\left\{p_{a}\right.$ : $a \in A$ or $a=0$, and $\left.p_{a} \neq 0\right\}$. $W$ is a partition of unity and there exists a unique $x \in \mathscr{R}$ such that $p_{a} \Vdash x=a$ for all $a \in A$ and $p_{0} \Vdash x=0$. Clearly, $x \geqslant a$ for all $a \in A$.

Proof of Axiom VII. If $A \subset \mathscr{R}$ such that $A$ has an upper bound, then

$\llbracket A$ has an upper bound $\rrbracket=1$,

and because

$$
\llbracket \text { every bounded set of reals has a least upper bound } \rrbracket=1 \text {, }
$$

there is an $a \in \mathscr{R}$ such that

$$
\llbracket a=\sup A \rrbracket=1,
$$

which, in turn, translates into $a=\sup A$.

Finally, we prove that the algebra of all projections in $\mathscr{A}$ is $\mathscr{B}$. We observe that

$$
\llbracket \text { if } a^{2}=a \text { then either } a=0 \text { or } a=1 \rrbracket=1,
$$

and therefore if $a \in \mathscr{A}$ is such that $a^{2}=a$, then

$$
\llbracket a=1 \rrbracket=p, \quad \llbracket a=0 \rrbracket=1-p
$$

for some $p \in \mathscr{B}$. However, $p$ itself is such that

$$
\llbracket p=1 \rrbracket=p \quad \text { and } \llbracket p=0 \rrbracket=1-p
$$

and, by uniqueness, $a=p$.

Thus we have

THEOREM C. For every complete Boolean algebra $\mathscr{B}$ there is a unique complete Stonean algebra $\mathscr{A}$ whose algebra of projections is $\mathscr{B}$; it is the algebra of $\mathscr{B}$-valued complex numbers.

We have the following correspondence:

\begin{tabular}{c|c}
$\mathscr{A}$ & $\mathbf{C}^{\mathscr{B}}$ \\
\hline 0,1 & 0,1 \\
$+, \cdot \cdot, \cdot$ \\
$*$ & complex conjugation \\
$x=\lambda \cdot 1$ & $\llbracket x=\lambda \rrbracket=1, \lambda \in \mathbf{C}$ \\
step function $s$ & $\llbracket s \in \mathbf{C} \rrbracket=1$ \\
reals & real numbers \\
$\leqslant$ & $\leqslant$ \\
projection $p$ & $\llbracket p \in\{0,1\} \rrbracket=1, p=\llbracket p=1 \rrbracket$ \\
$p a=p b$ & $p \Vdash a=b$ \\
$p a \leqslant p b$ & $p \Vdash a \leqslant b$
\end{tabular}


We note that the forcing relation $\Vdash$, as well as the Boolean values $\llbracket$, as we defined them for $=, \leqslant, \neq$ and $<$ in 2.19 and 2.21 , coincide with $\mathbb{\rrbracket}$ and $\Vdash$ in (4.6) and (4.7) under this correspondence.

We shall now turn our attention to convergence. Let $\left\{a_{n}\right\}$ be a net of elements of $\mathscr{A}$, and consider the statement

$$
\llbracket a=\lim a_{n} \rrbracket=1,
$$

or, equivalently.

$$
\llbracket \forall \varepsilon \in \mathbf{R}, \varepsilon>0, \exists N \forall a \geqslant N\left|a_{n}-a\right| \leqslant \varepsilon \rrbracket=1,
$$

which, when translated, gives

$$
\forall \varepsilon>0 \forall p \in P \exists q \in P, q \leqslant p, \exists N \forall n \geqslant N q \Vdash\left|a_{n}-a\right| \leqslant \varepsilon,
$$

and that, in turn, is equivalent to

$$
\begin{gathered}
(\forall \varepsilon>0)\left(\exists \text { partition of unity } W_{\varepsilon}\right) \exists\left\{N_{p}^{\varepsilon}: p \in W_{\varepsilon}\right\}\left(\forall p \in W_{\varepsilon}\right) \forall n \\
n \leqslant N_{p}^{\varepsilon} \text { implies }\left\|a p-a_{n} p\right\| \leqslant \varepsilon,
\end{gathered}
$$

and we have

4.20 Proposition. A net $\left\{a_{n}\right\} \subset \mathscr{A} \mathscr{B}$-converges to $a$ if and only if

$$
\llbracket \lim a_{n}=1 \rrbracket=1,
$$

which is why we defined $\mathscr{B}$-convergence in the first place.

5. Representation by continuous functions. We now use the Boolean-valued model to obtain a representation of complete Stonean algebras by algebras of continuous functions.

Let $\mathscr{A}$ be a complete Stonean algebra and let $\mathscr{B}$ be the algebra of all projections in $\mathscr{A}$. Let $\mathscr{X}$ be the Stone space [12] of $\mathscr{B}$, namely the set of all ultrafilters on $\mathscr{B}$, with the topology given by the basis of open sets

$$
U_{p}=\{x \in \mathscr{X}: p \in x\}
$$

The space $\mathscr{X}$ is compact and Hausdorff. The Boolean algebra $\mathscr{B}$ is isomorphic to the set algebra $\mathscr{B}^{\prime}$ of all clopen sets in $\mathscr{X}$; and because $\mathscr{B}$ is complete, is extremally disconnected (the closure of every open set is open, see [6]), and so $\mathscr{B}{ }^{\prime}$ is the collection of all regular open sets in $\mathscr{X}$.

Let

$$
\mathbf{C} \cup\{\infty\}, \quad \mathbf{R} \cup\{-\infty,+\infty)
$$

denote, respectively, the Riemann sphere (one-point-compactification of $\mathbf{C}$ ) and the extended real line. Following [5], we let

$$
\mathscr{N}(\mathscr{X})=\text { all continuous functions } f: \mathscr{X} \rightarrow \mathrm{C} \cup\{\infty\}
$$

such that $f_{-1}(\infty)$ is nowhere dense

(the normal functions on $\mathscr{X}$ ),

and

$$
\begin{aligned}
\mathscr{S}(\mathscr{X})= & \text { all continuous functions } f: \mathscr{X} \rightarrow \mathbf{R} \cup\{\infty,-\infty\} \\
& \text { such that } f_{-1}( \pm \infty) \text { is nowhere dense } \\
& (\text { the selfadjoint functions on } \mathscr{X}) .
\end{aligned}
$$


THEOREM B. Every complete Stonean algebra $\mathscr{A}$ is isomorphic to the algebra $\mathscr{N}(\mathscr{X})$, where $\mathscr{X}$ is the Stone space of the algebra $\mathscr{B}$ of all projections of $\mathscr{A}$. Under this isomorphism $B(\mathscr{A})$, the bounded part of $\mathscr{A}$, corresponds to $C(\mathscr{X})$, the algebra of all complex continuous functions on $\mathscr{X}$.

Proof. We identify $\mathscr{A}$ with the $\mathscr{B}$-valued complex numbers. For each $a \in \mathscr{R}=\mathbf{R}^{\mathscr{B}}$, let $f_{a}: \mathscr{X} \rightarrow \mathbf{R} \cup\{-\infty, \infty\}$ be as follows:

$$
f_{a}(x)=\inf \{\lambda \in \mathbf{R}: \llbracket a \leqslant \lambda \rrbracket \in x\} .
$$

First we show that each $f_{a}$ is continuous. Let $a \in \mathbf{R}$ and $x_{0} \in \mathscr{X}$. Assume that $f_{a}\left(x_{0}\right)=\lambda_{0} \in \mathbf{R}$ and let $\varepsilon>0$. It follows from (5.5) that $\llbracket a \leqslant \lambda_{0}+\varepsilon \rrbracket \in x_{0}$. Now, because

$$
\llbracket a \leqslant \lambda_{0}-\varepsilon \rrbracket+\llbracket a>\lambda_{0}-\varepsilon \rrbracket=1
$$

and the first term in (5.6) is not in $x_{0}$ (that would imply $f\left(x_{0}\right)<\lambda_{0}$ ), we have $\llbracket a>\lambda_{0}-\varepsilon \rrbracket \in x_{0}$, and so

$$
p=\llbracket \lambda_{0}-\varepsilon<a \leqslant \lambda_{0}+\varepsilon \rrbracket \in x_{0} .
$$

If $x \in U_{p}$, then, first, $\llbracket a \leqslant \lambda_{0}+\varepsilon \rrbracket \in x$ and we have $f_{a}(x) \leqslant \lambda_{0}+\varepsilon$. Second, for any $\lambda$ if $\llbracket a \leqslant \lambda \rrbracket \in x$, then $\lambda \geqslant \lambda_{0}-\varepsilon$, and therefore $f_{a}(x) \geqslant \lambda_{0}-\varepsilon$. Hence $f_{a}\left(U_{p}\right)$ $\subseteq\left[\lambda_{0}-\varepsilon, \lambda+\varepsilon\right]$.

Assume that $f_{a}(x)=+\infty$ and let $\lambda_{0} \in \mathbf{R}$. Since $\llbracket a>\lambda \rrbracket \in x_{0}$ for every $\lambda \in \mathbf{R}$, we let $p=\llbracket a>\lambda_{0} \rrbracket$ and it follows that $f_{a}\left(U_{p}\right) \subseteq\left[\lambda_{0},+\infty\right]$.

Finally, assume that $f_{a}\left(x_{0}\right)=-\infty$ and let $\lambda_{0} \in \mathbf{R}$. We have $\llbracket a \leqslant \lambda \rrbracket \in x_{0}$ for every $\lambda \in \mathbf{R}$, and so if we let $p=\llbracket a \leqslant \lambda_{0} \rrbracket$, we have $f_{a}\left(U_{p}\right) \subseteq\left[-\infty, \lambda_{0}\right]$.

It is easy to see that $f_{a}$ is finite on an open dense set. As the topology is given by the sets $U_{p}$, this amounts to the fact that

$$
\begin{aligned}
& \text { for every } p \in P \text { there exists } q \in P, q \leqslant p, \text { and } \lambda \in \mathbf{R} \\
& \text { such that } q \Vdash|a| \leqslant \lambda .
\end{aligned}
$$

(Then $f_{a}(x) \in[-\lambda, \lambda]$ for all $x \in U_{q}$.)

It is not difficult to verify that the correspondence

$$
a \mapsto f_{a}
$$

respects $\leqslant,+$ and $\cdot$. To verify that the correspondence (5.8) is one-to-one, it suffices to show that $f_{a} \neq 0$ whenever $a>0$. But if $a>0$, then there is a $p \in P$ and an $\varepsilon>0$ such that $p \Vdash a \geqslant \varepsilon$. It follows that for every $x \in U_{p}, f_{a}(x) \geqslant \varepsilon$, and so $f_{a} \neq 0$.

It remains to show that the correspondence (5.8) is between $\mathscr{A}$ and all functions in $\mathscr{N}(\mathscr{X})$. Let $f \in \mathcal{N}(\mathscr{X})$. For each $\lambda \in \mathbf{R} \cup\{-\infty, \infty\}$, we let

$$
F_{\lambda}=\{x \in \mathscr{X}: f(x) \leqslant \lambda\},
$$

and let $E_{\lambda}$ be the closure of the interior of $F_{\lambda}$. For each $\lambda$ we have $F_{\lambda}=\bigcap_{\varepsilon>0} F_{\lambda+\varepsilon}=$ $\cap\left\{F_{\mu}: \mu>\lambda\right.$ rational $\}$. As we identified regular open sets in $\mathscr{X}$ with elements of $\mathscr{B}$, we see that $\left\langle E_{\lambda}: \lambda \in \mathbf{R}\right\rangle$ has properties (4.2). Thus let $\mathscr{A}$ be

$$
a=\int \lambda d E_{\lambda}=\sup \left\{\lambda E_{\lambda}: \lambda \in \mathbf{R}\right\}
$$


We claim that $f_{a}=f$. This is proved as follows: For each rational $\lambda \in \mathbf{R} \cup\{-\infty, \infty\}$, let $N_{\lambda} \subseteq \mathscr{X}$ be the nowhere dense set $\left(E_{\lambda}-F_{\lambda}\right) \cup\left(F_{\lambda}-E_{\lambda}\right)$, and let

$$
M=\bigcup_{\lambda \text { rational }} N_{\lambda} \text {. }
$$

The set $M$ is meager and, for every $x \in \mathscr{X}-M$ and every rational $\lambda$,

$$
x \in E_{\lambda} \quad \text { iff } \quad x \in E_{\mu} \text { for all rational } \mu>\lambda .
$$

It follows that, for every $x \in \mathscr{X}-M$ and every rational $\lambda$,

$$
f(x) \leqslant \lambda \text { iff } f_{a}(x) \leqslant \lambda .
$$

As the rational numbers are dense in $\mathbf{R}$, we have

$$
f(x)=f_{a}(x)
$$

for all $x$ in $\mathscr{X}-M$, but since $\mathscr{X}-M$ is dense in $\mathscr{X}$, and $f$ and $f_{a}$ are continuous, we have $f=f_{a}$.

We have not really proved that bounded elements of $\mathscr{A}$ correspond to finite continuous functions on $\mathscr{X}$ but that should be obvious, granting the fact that

$$
\|f\|=\sup _{x \in \mathscr{X}}|f(x)| \quad \text { for any } f \in \mathscr{N}(\mathscr{X}) .
$$

Thus uniform convergence in $\mathscr{N}(\mathscr{X})$ is just uniform convergence of functions. As for the other two kinds of convergence, we have

5.10 TheOREM. (a) $A$ net $\left\{f_{n}\right\}$ in $\mathscr{N}(\mathscr{X})$ converges to $f$ piecewise uniformly if and only if there is an open dense set $U$ such that $f_{n}(x) \rightarrow f(x)$ for all $x \in U$.

(b) A countable set $\left\{f_{n}\right\}$ in $\mathscr{N}(\mathscr{X}) \mathscr{B}$-converges to $f$ if and only if there is a comeager set $C$ such that $f_{n}(x) \rightarrow f(x)$ for all $x \in C$.

[One direction of (b) holds for nets in general.]

Proof. (a) Let $f_{n} \rightarrow f$ piecewise uniformly. There is a partition of unity $W$ such that each $E \in W$ is a clopen set and $f_{n} \rightarrow f$ uniformly on $E$. Then $f_{n}(x)$ converges to $f(x)$ for all $x$ in the dense open set $U\{E: E \cap W\}$.

Conversely, let $U$ be an open dense set such that $f_{n}(x) \rightarrow f(x)$ for all $x \in U$. There exists a partition of unity $W$ such that each $E \in W$ is clopen and $E \subseteq U$. Since $\mathscr{X}$ is compact, $f_{n}$ converges to $f$ uniformly on each $E \in W$. Hence $f_{n} \rightarrow f$ piecewise uniformly.

(b) Let $\left\{f_{n}\right\}$ be a net and let $f$ be a $\mathscr{B}$-limit of $f_{n}$. For each $k$ there is a partition of unity $W_{k}$ and a set $\left\{N_{p}^{k}: p \in W_{k}\right\}$ such that $\left|f_{n}-f\right| \leqslant 1 / k$ on $p$ for all $n \geqslant N_{p}$. Let $U_{k}=\bigcup\left\{p: p \in W_{k}\right\}$ and $C=\bigcap_{k=1}^{\infty} U_{k} . C$ is a comeager set and, for all $x \in C$ and every $k$, there is an $N$ such that $\left|f_{n}(x)-f(x)\right| \leqslant 1 / k$ for all $n \geqslant N$. Hence $f_{n}(x) \rightarrow f(x)$ on $C$.

Now let $\left\{f_{n}\right\}$ be a countable net and assume that $f_{n}$ does not $\mathscr{B}$-converge to $f$. Hence $\llbracket f=\lim f_{n} \rrbracket \neq 1$, and so

$$
(\exists \varepsilon>0) \exists p_{0} \forall N \forall p \leqslant p_{0} \exists q \leqslant p \exists n \geqslant N \text { such that } q \Vdash\left|f_{n}-f\right| \geqslant \varepsilon .
$$

Let $\varepsilon \in 0$ and $p_{0} \in P$ be given by (5.11); (5.11) can be reformulated as follows:

$$
\begin{aligned}
& \forall N\left(\exists \text { partition } W_{n} \text { of } p_{0}\right)\left(\left\{n_{p}^{N}: p \in W_{N}\right\}\right) \text { such that } \\
& n_{p}^{N} \geqslant N \text { and } p \Vdash\left|f_{n_{p}^{N}}-f\right| \geqslant \varepsilon \text { for all } p \in W_{N} .
\end{aligned}
$$


For each $N$ let $U_{N}=\bigcup\left\{p: p \in W_{N}\right\}$ and let $S=\bigcap_{N=1}^{\infty} U_{N}$. The set $S$ is comeager on $p_{0}$ and, for each $x \in S$, there are cofinally many $n$ such that $\left|f_{n}(x)-f(x)\right| \geqslant \varepsilon$. Hence $f_{n}(x)$ does not converge to $f(x)$ for all $x$ in a set $S$ that is comeager on a nonempty open set.

6. Function calculus. In this section we prove Theorem D.

Theorem D. Let $\mathscr{A}$ be a complete Stonean algebra and let $a \in \mathscr{A}$. There is a homomorphism $\varphi_{a}$ of the algebra of Borel functions $f: \mathbf{C} \rightarrow \mathbf{C}$ into $\mathscr{A}$ such that:

(a) $\varphi_{a}$ (id) $=a$, and

(b) if $f_{n}(x) \rightarrow f(x)$ pointwise, then $\varphi(f)$ is a $\mathscr{B}$-limit of $\varphi\left(f_{n}\right)$.

[The theorem can be extended to functions of several variables.]

We identify $\mathscr{A}$ again with the $\mathscr{B}$-valued complex numbers, where $\mathscr{B}$ is the algebra of projections in $\mathscr{A}$.

The set of all Borel functions $f: \mathbf{C} \rightarrow \mathbf{C}$ is the smallest collection of functions that contains all continuous functions and is closed under taking pointwise limits of sequences. The functions form a heirarchy of length $\omega_{1}$, where continuous functions are of class 0 and functions of class $\alpha$ are limits of sequences of functions of class $<\alpha$. Each continuous function can be coded by a single (infinite) $0-1$ sequence, and the process of generating Borel functions of higher classes can also be so coded. Consequently, questions on Borel functions are encoded as properties of certain 0-1 sequences. The set of all codes of Borel functions (as well as sequences of Borel functions) is a $\Pi_{1}^{1}$ set (i.e. coanalytic), and simple properties of Borel functions, such as $(\forall x) \lim f_{n}(x)=f(x)$, are $\Pi_{1}^{1}$ relations between their codes. A detailed description of coding Borel sets can be found e.g. in [10, or 4, §42]; the codes of Borel functions are defined similarly.

It is a standard fact of models of set theory that $\Pi_{1}^{1}$ relations are absolute for Boolean-valued models. Thus if $f$ is a Borel function on $\mathbf{C}$ and $c$ is its code, then in $V^{\mathscr{B}}$ it holds that $c$ is a code of a Borel function (on $\mathbf{C}^{\mathscr{B}}$ ); we denote this function by $f^{\mathscr{B}}$. By absoluteness, if $\lambda \in \mathbf{C}$, then $f^{\mathscr{B}}(\lambda)=f(\lambda)$, and so $f^{\mathscr{B}}$ is an extension of $f$.

The correspondence $f \mapsto f^{\mathscr{B}}$ has, among others, the following properties:

(a) if $f$ is a constant function $\lambda$, then $f^{\mathscr{B}}$ is a constant function $\lambda$,

(b) if $f$ is the identity function, then $f^{\mathscr{D}}$ is the identity function,

(c) $(f+g)^{\mathscr{D}}=f^{\mathscr{B}}+g^{\mathscr{B}},(f \cdot g)^{\mathscr{B}}=f^{\mathscr{B}} \cdot g^{\mathscr{B}}$ and $\left(f^{*}\right)^{\mathscr{B}}=\left(f^{\mathscr{B}}\right)^{*}$,

(d) if $f \leqslant g$, then $f^{\mathscr{B}} \leqslant g^{\mathscr{B}}$,

(e) if $(\forall x \in \mathbf{C}) f(x)=\lim f_{n}(x)$, then $\left(\forall x \in \mathbf{C}^{\mathscr{B}}\right) \llbracket f^{\mathscr{D}}(x)=\lim f_{n}^{\mathscr{D}}(x) \rrbracket=1$.

This is because all the properties in question are absolute for the Boolean-valued model $V^{\mathscr{B}}$.

Now let $a$ be a fixed element of $\mathscr{A}=\mathbf{C}^{\mathscr{B}}$. We define the homomorphism $\varphi_{a}$ as follows:

$$
\varphi_{a}(f)=f^{\mathscr{D}}(a)
$$


for every Borel function $f: \mathbf{C} \rightarrow \mathbf{C}$. The previous discussion confirms that $\varphi_{a}$ is a homomorphism. By (6.1)(b),

$$
\varphi_{a}(\mathrm{id})=\mathrm{id}^{\mathscr{B}}(a)=a
$$

and, by $(6.1)(\mathrm{e})$, if $f(x)=\lim f_{n}(x)$ for all $x$, then

$$
\llbracket f(a)=\lim f_{n}^{\mathscr{B}}(a) \rrbracket=1,
$$

and so $\varphi_{a}(f)$ is the $\mathscr{B}$-limit of $\left\{\varphi_{a}\left(f_{n}\right)\right\}$.

7. Completion of a Stonean algebra. In this section we prove Theorem A.

7.1 Definition. Let $\mathscr{A}$ be a Stonean algebra. A complete Stonean algebra $\bar{A}$ is a completion of $\mathscr{A}$ if $B(\mathscr{A})$ is (isomorphic to) a dense subalgebra of $B(\bar{A})$.

THEOREM A. Every Stonean algebra has a unique completion.

7.2. The uniqueness part of Theorem $\mathrm{A}$ follows from Lemma 2.30. If $\mathscr{A}_{1}$ and $\mathscr{A}_{2}$ are both completions of $\mathscr{A}$, then

$$
\pi(\mathscr{A})=\sup _{\mathscr{A}_{2}}\{x \in \mathscr{A}: x \leqslant a\} \quad\left(a \in \mathscr{A}_{1}\right)
$$

is an isomorphism between $\mathscr{A}_{1}$ and $\mathscr{A}_{2}$.

7.3. Let $\mathscr{A}$ be a Stonean algebra. We are going to construct a completion $\overline{\mathscr{A}}$ of $\mathscr{A}$ by constructing the complete Boolean algebra of projections of $\overline{\mathscr{A}}$ and then embed$\operatorname{ding} \mathscr{A}$ in the (uniquely determined) $\mathscr{A}$. We remark that $\mathscr{B}$ is, in general, not the completion of the algebra of projections of $\mathscr{A}$. For instance, the Stonean algebra $C[0,1]$ has only two projections, 0 and 1 , but its completion has an infinite algebra of projections.

7.4 Definition. Let $Q$ be the set of all positive elements of $\mathscr{A}$. Two elements $u, v$ of $Q$ are orthogonal, $u \perp v$ if $u \cdot v=0$.

On $Q$ we define the relation $\preccurlyeq$ as follows:

$$
u \preccurlyeq v \quad \text { if, for every } w \in Q, w \perp V \text { implies } w \perp u \text {. }
$$

The relation $\preccurlyeq$ is transitive, and so let

$$
u \simeq v \text { if } u \preccurlyeq v \text { and } v \preccurlyeq u .
$$

7.7 Definition. Let $P$ be the set of all equivalence classes of $Q$. The elements of $P$ are conditions, and on $P$ we define

$$
p \leqslant q(p \text { is stronger than } q) \text { if } u \leqslant v(u \in p, v \in q) ;
$$

$(P,<)$ is a partial ordering.

Two conditions are orthogonal $p \perp q$ if $u \perp v$ for some (any) $u \in p, v \in q$.

7.8 LemMA. (a) If $u, v \in Q$ and $u \cdot v \neq 0$, then $u v \preccurlyeq u$ and $u v \preccurlyeq v$.

(b) $u \perp u$ for no $u \in Q$.

Proof. (a) is obvious; (b) is Axiom II(d).

7.9 Lemma. For every $p, q \in P, p \perp q$ if and only if there is no $r \in P$ such that $r \leqslant p$ and $r \leqslant q$.

Proof. In other words, $u \cdot v \neq 0$ iff there is a $w$ such that $w \preccurlyeq u$ and $w \leqslant v$. If $u v \neq 0$, then let $w=u v$. 
Conversely, let $u v=0$ and assume that $w$ is such that $w \preccurlyeq u$ and $w \preccurlyeq v$. First, because $w \preccurlyeq u$ and $v \perp u$, we have $v \perp w$. Second, because $w \preccurlyeq v$ and $w \perp v$, we have $w \perp w$, a contradiction.

7.10 LEMMA. If $p$ is not stronger than $q$, then there exists an $r \in P$ such that $r \leqslant p$ and $r \perp q$.

Proof. If $u \nless v$, then there exists a $w$ such that $w v=0$ and $w u \neq 0$. Hence $w u \preccurlyeq u$ and $w u \perp v$.

7.11 Definition. A partially ordered set $(P,<)$ is separative if it has the property stated in Lemma 7.10.

It is well known (see $[4, \S 17]$ ) that every separative partially ordered set is densely embedded in a unique complete Boolean algebra $\mathscr{B}$. Thus we let $\mathscr{B}$ be the complete Boolean algebra that has the partial order $(P,<)$ defined above as a dense set.

7.12 Definition. For $a \in \mathscr{R}$, let $a^{+}=a \vee 0, a^{-}=a \wedge 0$.

7.13 LEMMA. (a) $a=a^{+}+a^{-}$,

(b) $a \geqslant 0$ iff $a^{+}=a$ iff $a^{-}=0$, and

(c) if $u \geqslant 0$, then $(u a)^{+}=u a^{+}$and $(u a)^{-}=u a^{-}$.

Proof. Axiom II(e), (f).

7.14 Definition. Let $u \in Q, a, b \in \mathscr{R}$ :

$$
u \Vdash a=b \quad \text { if } u a=u b, \quad u \Vdash a \leqslant b \quad \text { if } u a \leqslant u b .
$$

(We say that $u$ forces $a=b, u$ forces $a \leqslant b$.)

7.15 LEMMA. $u \Vdash a \leqslant 0$ if and only if $u \Vdash a^{+}=0$.

Proof. By Lemma 7.13.

7.16 LEMMA. (a) If $v \preccurlyeq u$ and $u \Vdash a=b$, then $v \Vdash a=b$.

(b) If $v \preccurlyeq u$ and $u \Vdash a \leqslant b$, then $v \Vdash a \leqslant b$.

Proof. (a) We may assume that $b=0$. If $u \Vdash a=0$, then $u a^{+}=0$ and $u a^{-}=0$. If $a^{+}=0$, then $v a^{+}=0$, and we are done. If $a^{+}>0$, then $a^{+} \in Q$ and, because $a^{+} \perp u$ and $v \preccurlyeq u$, we have $a^{+} \perp v$, and so $v a^{+}=0$. Similarly, $v a^{-}=0$, and so $v a=0$.

(b) This follows from (a) and Lemma 7.15.

7.17 Definition. Let $u \in Q, a, b \in \mathscr{R}$.

$$
\begin{array}{ll}
u \Vdash a \neq b & \text { if no } v \leqslant u \text { forces } a=b, \\
u \Vdash a<b & \text { if } u \Vdash a \leqslant b \text { and } u \Vdash a \neq b .
\end{array}
$$

7.18 LEMMA. (a) If $v \preccurlyeq u$ and $u \Vdash a \neq b$, then $v \Vdash a \neq b$.

(b) If $v \preccurlyeq u$ and $u \Vdash a<b$, then $v \Vdash a<b$.

Proof. (a) From definition and transitivity of $\preccurlyeq$.

(b) From (a) and Lemma 7.16(b).

7.19 LemMA. $u \Vdash u>0$ for every $u \in Q$. 
Proof. Clearly, $u \Vdash u \geqslant 0$. To show that $u \Vdash u>0$, we have to show that no $v \preccurlyeq u$ forces $u=0$. Let $v \preccurlyeq u$ and assume that $v \Vdash u=0$. Because $v \perp u$ and $v \preccurlyeq u$, we have $v \perp v$, a contradiction.

7.20 LemMA. Let $u \in Q, a, b \in \mathscr{R}$.

(a) $u \Vdash a \neq b$ iff there is no $v \preccurlyeq u$ such that $v \Vdash a=b$,

(b) $u \Vdash a=b$ iff there is no $v \preccurlyeq u$ such that $v \Vdash a \neq b$,

(c) $u \Vdash a \leqslant b$ iff there is no $v \preccurlyeq u$ such that $v \Vdash a>b$, and

(d) $u \Vdash a<b$ iff there is no $v \preccurlyeq u$ such that $v \Vdash a \geqslant b$.

Proof. The implications from left to right are obvious, as no $v$ simultaneously forces $a=b$ and $a \neq b$, etc. Besides, we may assume that $b=0$.

(a) This is just Definition 7.17.

(b) First we observe that if $u \Vdash a^{+}=0$ and $u \Vdash a^{-}=0$, then $u \Vdash a=0$. Hence we may assume that $a \geqslant 0$, and if $a=0$, then there is nothing to prove. Thus let $a>0$ and assume that $u$ does not force $a=0$; hence $u a \neq 0$. By Lemma 7.19, $a \Vdash a>0$ and, because $v=u a \preccurlyeq a$, we have, by Lemma 7.18(a), that $v \Vdash a>0$. But $v \preccurlyeq u$.

(c) Assume that $u$ does not force $a \leqslant 0$. Then $u a \nless 0$, and by Axiom III there is a $v>0$ such that $v u a>0$. Hence $v u \in Q$ and $v u \Vdash a \geqslant 0$. Since $v u a \neq 0$, $v u$ does not force $a=0$. By (b), there is a $w \leqslant v u$ such that $w \Vdash a \neq 0$ and, by Lemma 7.16(b), $w \Vdash a \geqslant 0$. Hence $w \Vdash a>0$.

(d) Assume that $u$ does not force $a<b$. Either $u$ does not force $a \leqslant b$, in which case there is a $v \preccurlyeq u$ (by (c)) such that $v \Vdash a>b$, hence $v \Vdash a \geqslant b$. Or else $u$ does not force $a \neq b$ in which case there is a $v \preccurlyeq u$ (by (a)) such that $v \Vdash a=b$; hence again $v \Vdash a \geqslant b$.

7.21 Definition. Boolean values. For $a, b \in \mathscr{R}$, we define (in $\mathscr{B}$ )

$$
\begin{array}{ll}
\llbracket a=b \rrbracket=\bigvee\{p: p \Vdash a=b\}, & \llbracket a \leqslant b \rrbracket=\bigvee\{p: p \Vdash a \leqslant b\}, \\
\llbracket a \neq b \rrbracket=\bigvee\{p: p \Vdash a \neq b\}, & \llbracket a<b \rrbracket=\bigvee\{p: p \Vdash a<b\} .
\end{array}
$$

As $\Vdash$ is invariant under the equivalence (7.6), “ $p \Vdash$ " makes sense. The operation $V$ is the Boolean-algebraic supremum. The Boolean algebra $\mathscr{B}$ contains $P$ as a subset.

7.22 LEMMA. (a) $\llbracket a \neq b \rrbracket=1-\llbracket a=b \rrbracket$,

(b) $\llbracket a<b \rrbracket=\llbracket a \leqslant b \rrbracket \cdot \llbracket a \neq b \rrbracket$,

(c) $\llbracket a=b \rrbracket=\llbracket a \leqslant b \rrbracket \cdot \llbracket a \geqslant b \rrbracket$,

(d) $\llbracket a<b \rrbracket=1-\llbracket a \geqslant b \rrbracket$.

Proof. Lemmas 7.16 and 7.18 through 7.20.

We shall embed $\mathscr{A}$ into the $\mathscr{B}$-valued complex numbers $C^{\mathscr{B}}$. We need two more lemmas first.

7.23 LEMmA. Let $a \in \mathscr{R}$. If $p$ does not force $a \leqslant 0$, then there exists $q \leqslant p$ and $\varepsilon>0$ such that $q \Vdash a \geqslant \varepsilon$. 
Proof. Let $u \in Q$ and assume that $u$ does not force $a \leqslant 0$. Then there is a $v \leqslant u$ such that $v \Vdash a>0$. Let $w=v \wedge 1$. By Axiom $\mathrm{II}(\mathrm{g}), w>0$, and clearly $w \preccurlyeq v$; hence $w \Vdash a>0$.

As $w a \nless 0$, there is, by Axiom IV, some $\varepsilon>0$ such that $w a \nless \varepsilon$. Since $w \leqslant 1$, we have $\varepsilon w \leqslant \varepsilon$, and so $w a \nless \varepsilon$. Hence $w$ does not force $a \leqslant \varepsilon$, and by Lemma 7.20(c) there is a $z \preccurlyeq w$ such that $z \Vdash a>\varepsilon$.

7.24 Corollary. For $a \in \mathscr{R}$ and $\lambda \in \mathbf{R}$,

$$
\llbracket a \leqslant \lambda \rrbracket=\bigwedge_{\varepsilon>0} \llbracket a \leqslant \lambda+\varepsilon \rrbracket .
$$

7.25 Lemma. Let $a \in \mathscr{R}$. For every $p$ there is $a q \leqslant p$ and some $\lambda \in \mathbf{R}$ such that $q \Vdash a \leqslant \lambda$.

Proof. Let $u \in Q$; we may assume that $a \geqslant 0$. By Axiom $\mathrm{V}$ there is a $v$, $0<v \leqslant u$, and some $\mu \in \mathbf{R}$ such that $v a \leqslant \mu$. By Lemma 7.19, $v \Vdash v>0$. By Lemma 7.23 there is a $w \leqslant v$ and an $\varepsilon>0$ such that $w \Vdash v \geqslant \varepsilon$; hence $\varepsilon w \leqslant w v$. Hence $\varepsilon w a \leqslant w v a \leqslant \mu a$, and so $w \Vdash a \leqslant \lambda$.

7.26 Corollary. Let $a \in \mathscr{R}$ :

$$
\bigwedge_{\lambda \in \mathbf{R}} \llbracket a \leqslant \lambda \rrbracket=0, \quad \bigvee_{\lambda \in \mathbf{R}} \llbracket a \leqslant \lambda \rrbracket=1
$$

7.27 Definition. For every $a \in \mathscr{R}$, let $\pi(a) \in \mathbf{R}^{\mathscr{P}}$ be the unique $\mathscr{B}$-valued real a such that

$$
\llbracket \mathbf{a} \leqslant \lambda \rrbracket=\llbracket a \leqslant \lambda \rrbracket \quad(\text { all } \lambda \in \mathbf{R}),
$$

i.e.

$$
\pi(a)=\int \lambda d \llbracket a \leqslant \lambda \rrbracket .
$$

By Corollaries 7.24 and 7.26, the Boolean values $\llbracket a \leqslant \lambda \rrbracket$ satisfy the conditions (4.2) and so $\pi$ is well defined.

7.28 Lemma. For all $a, b \in \mathscr{R}$ and $\lambda \in \mathbf{R}$,

$$
\begin{gathered}
\text { if } a \leqslant b, \text { then } \llbracket \pi(a) \leqslant \pi(b) \rrbracket=1, \\
\llbracket \pi(a)+\pi(b)=\pi(a+b) \rrbracket, \\
\llbracket \pi(a) \cdot \pi(b)=\pi(a \cdot b) \rrbracket=1, \\
\llbracket \pi(\lambda a)=\lambda \pi(a) \rrbracket, \\
\llbracket \pi(0)=0 \rrbracket=1, \quad \llbracket \pi(1)=1 \rrbracket=1 .
\end{gathered}
$$

Proof. Exercise in Boolean-valued calculus.

7.29 LEMMA. If $a \neq b$, then $\pi(a) \neq \pi(b)$.

Proof. It suffices to show that if $a \neq 0$, then $\pi(a) \neq 0$. If $a \neq 0$, then there is a $u>0$ such that $u \Vdash a \neq 0$, and so $\llbracket a=0 \rrbracket \neq 1$. By the definition of $\pi, \llbracket \pi(a)=0 \rrbracket$ $=\llbracket a=0 \rrbracket$ (where the left Boolean value is defined in $V^{\mathscr{B}}$ and the right Boolean value is the one defined in 7.21), and because $\llbracket \pi(0)=0 \rrbracket=1$, we have $\pi(a) \neq \pi(0)$. 
It remains to prove that $\pi$ maps the bounded elements of $\mathscr{R}$ onto a dense subset of the bounded part of $\mathbf{R}^{\mathscr{D}}$. As the step functions are dense in $\mathbf{R}^{\mathscr{B}}$, and $P$ is a dense subset of $\mathscr{B}$, it suffices to prove the following lemma.

7.30 Lemma. For every $p \in P$ and every $\varepsilon>0$ there is an $a \in \mathscr{A}$ such that $0<a \leqslant \varepsilon p$.

Proof. Let $p \in P$ and $\varepsilon>0$. Let $u>0$ be such that $u \in p$. Let $v=u \wedge \varepsilon$. By Axiom $\mathrm{II}(\mathrm{g})$ we have $v>0$. As $v \leqslant \varepsilon$, we have $\llbracket \pi(v) \leqslant \varepsilon \rrbracket=1$. Every condition orthogonal to $u$ forces that $v=0$, and so $1-p \leqslant \llbracket \pi(v)=0 \rrbracket$. Now, because $\llbracket \varepsilon p=\varepsilon \rrbracket=p$ and $\llbracket \varepsilon p=0 \rrbracket=1-p$, we have $\llbracket \pi(v) \leqslant \varepsilon p \rrbracket=1$, and so $\pi(v) \leqslant \varepsilon p$.

We end this section with an example.

7.31. Let $\mathscr{A}=C[0,1]$. If $u>0$ and $v>0$ are continuous functions on $[0,1]$, then $u \leqslant v$ just in case $u(t)>0$ implies $v(t)>0$. Hence $\mathscr{B}$ is the completion of the partially ordered set of all open subsets of $[0,1]$ (ordered by inclusion). That is, $\mathscr{B}$ is the Boolean algebra of Borel sets modulo meager set or, equivalently, the algebra of all regular open subsets $[0,1]$, and the completion of $\mathscr{A}$ is the complete Stonean algebra with projections $\mathscr{B}$.

8. More on convergence and completeness. In $\S 3$ we proved that if a Stonean algebra $\mathscr{A}$ is complete, then every $\mathscr{B}$-Cauchy net has a $\mathscr{B}$-limit. We shall now prove the converse. Then we discuss complete subalgebras of complete Stonean algebras.

8.1 Theorem. Let $\mathscr{A}$ be the completion of a Stonean algebra $\mathscr{A}_{0}$. Then every $a \in \mathscr{A}$ is the $\mathscr{B}$-limit of a net in $\mathscr{A}_{0}$.

Proof. Every $a \in \mathscr{A}$ has the form $x+i y$ where $x, y \in \mathscr{R}$, and every $a \in \mathscr{R}$ is $a \cdot \llbracket a \geqslant 0 \rrbracket+a \cdot \llbracket a \leqslant 0 \rrbracket$; hence it suffices to show that every $a \geqslant 0$ is the $\mathscr{B}$-limit of a net in $\mathscr{A}_{0}$. For every $\varepsilon \geqslant 0$ let

$A_{\varepsilon}=$ a maximal set of $u \in \mathscr{A}_{0}, 0<u \leqslant a$, such that the projections $p_{u}^{\varepsilon}=\llbracket a-u \leqslant \varepsilon \rrbracket$ are positive and mutually orthogonal.

\subsection{LeMMA. For each $\varepsilon>0$, the set $A_{\varepsilon}$ is a partition of unity.}

Proof. We are to show that $\vee A_{\varepsilon}=1$. Thus let us assume, for contradiction, that there is a condition $p$, orthogonal to each $p_{u}^{\varepsilon}, u \in A_{\varepsilon}$. There is a stronger condition $q$ and numbers $\nu_{1}<\nu_{2}$ such that $q \Vdash a-\varepsilon \leqslant \nu_{1}$ and $q \Vdash \nu_{2} \leqslant a$. Let $v \in \mathscr{A}_{0}$ be such that $v \preccurlyeq q$; we may also assume that $v$ is bounded. Let $\lambda=\|v\|$ and $u=\nu_{2} v / \lambda$. Then $u \leqslant q, u \leqslant \nu_{2}$ and therefore $u \leqslant a$; also, $\left\|u \geqslant \nu_{1}\right\|>0$ and so $p_{u}^{\varepsilon}=\llbracket a-\varepsilon \leqslant u \rrbracket>0$. This contradicts the maximality of $A_{\varepsilon}$.

8.4. Now we can finish the proof of Theorem 8.1. Let $A=\cup_{\varepsilon>0} A_{\varepsilon}$ and let $D$ be the set of all finite subsets $E$ of $A$, ordered by inclusion. $D$ is a directed set.

For each $E=\left\{u_{1}, \ldots, u_{n}\right\} \in D$, let $a_{E}=u_{1} \vee \cdots \vee u_{n}$. Then $a$ is a $\mathscr{B}$-limit of the net $\left\{a_{E}\right\}$, because for each $\varepsilon>0$ there is a partition $\left\{p_{u}^{\varepsilon}: u \in A_{\varepsilon}\right\}$ and $\left\{E_{u}^{\varepsilon}\right.$ : $\left.u \in A_{\varepsilon}\right\}$, namely $E_{u}^{\varepsilon}=\{u\}$, which attests to $\mathscr{B}$-lim $\lim _{E} a_{E}=a$. 
8.5 Definition. A Stonean algebra is $\sigma$-saturated if there exists no uncountable set $A$ such that $a \cdot b=0$ for all distinct $a, b \in A$.

8.6 Lemma. A Stonean algebra is o-saturated if and only if its completion is $\sigma$-saturated. A complete Stonean algebra is $\sigma$-saturated if and only if its Boolean algebra of projections is $\sigma$-saturated.

In case of Boolean algebras, this property is generally referred to as the countable chain condition [9].

8.7 TheOREM. Let $\mathscr{A}$ be the completion of a $\sigma$-saturated Stonean algebra $\mathscr{A}_{0}$. Then every $a \in \mathscr{A}$ is the $\mathscr{B}$-limit of a sequence in $\mathscr{A}_{0}$.

Proof. Let $A_{\varepsilon}$ be as in the proof of Theorem 8.1. Since $\mathscr{A}$ is $\sigma$-saturated, each $A_{\varepsilon}$ is countable. Let $A=\bigcup_{k=1}^{\infty} A_{1 / k}$, let $\left\{u_{n}\right\}$ be any enumeration of $A$, and let $a_{n}=u_{1} \vee$ $\cdots \vee u_{n}$. Then $a=\mathscr{B}-\lim _{n} a_{n}$.

We shall now characterize completeness in terms of convergence of Cauchy nets. Let $\mathscr{A}$ be a Stonean algebra, not necessarily complete. The definition of $\mathscr{B}$-convergence given in $\S 3$ is not well suited in this case, as $\mathscr{A}$ may have no nontrivial projections. Thus we first give a more general definition of $\mathscr{B}$-convergence that is applicable even if $\mathscr{A}$ is not complete.

8.8 Definition. A partition of unity is a maximal set $W$ of positive reals such that $u \cdot v=0$ whenever $u, v \in W$ and $u \neq v$.

8.9 Definition. $a$ is a $\mathscr{B}$-limit of a net $\left\{a_{n}\right\}$ if for every $\varepsilon>0$ there is a partition of unity $W_{\varepsilon}$ and $\left\{N_{u}^{\varepsilon}: u \in W\right\}$ such that for all $u \in W$

$$
\left|u a-u a_{n}\right| \leqslant \varepsilon u \quad \text { whenever } n \geqslant N_{u}^{\varepsilon} \text {. }
$$

(If $\mathscr{A}$ is complete, then the definition agrees with the definition in $\S 3$.) In terms of forcing, we have

8.10 LEMMA. $a=\mathscr{B}$ - $\lim a_{n}$ if and only if

$$
\forall \varepsilon>0, \quad \forall p \in P \exists N \exists q \leqslant p q \Vdash\left|a-a_{n}\right| \leqslant \varepsilon .
$$

8.11 TheOREM. A Stonean algebra $\mathscr{A}$ is complete if and only if every $\mathscr{B}$-Cauchy net has a $\mathscr{B}$-limit.

Proof. If $\mathscr{A}$ is complete, then every $\mathscr{B}$-Cauchy net is $\mathscr{B}$-convergent by Lemma 3.5(c). Conversely, let $\mathscr{A}_{0}$ be a Stonean algebra. Let $\mathscr{A}$ be the completion of $\mathscr{A}_{0}$, so by Theorem 8.1 every $a \in \mathscr{A}$ is the $\mathscr{B}$-limit of a net in $\mathscr{A}_{0}$. However, the $\mathscr{B}$-convergence in Theorem 8.1 refers to the $\mathscr{B}$-convergence defined in terms of the Boolean algebra $\mathscr{B}$ of projections in $\mathscr{A}$. If we prove that $\mathscr{B}$ is included in $\mathscr{A}_{0}$, then every net in $\mathscr{A}_{0}$ that is $\mathscr{B}$-Cauchy in $\mathscr{A}$ is $\mathscr{B}$-Cauchy in $\mathscr{A}_{0}$, and hence its $\mathscr{B}$-limit is in $\mathscr{A}_{0}$, by the assumption of the theorem. That will prove that $\mathscr{A}_{0}=\mathscr{A}$ and so $\mathscr{A}_{0}$ is complete.

We wish to show that $\mathscr{B} \subseteq \mathscr{A}_{0}$. Thus let $a \in \mathscr{B}$. We construct the net $\left\{a_{E}\right\}$ in $\mathscr{A}_{0}$ as in the proof of Theorem 8.1. In particular, we define $A_{\varepsilon}$ by (8.2). The important point is, however, that the projection $\llbracket a-u \leqslant \varepsilon \rrbracket$ is in $P$ : it is the equivalence class of

$$
v_{u}^{\varepsilon}=(u-(1-\varepsilon)) \vee 0,
$$


a positive real in $\mathscr{A}_{0}$. We have $v_{u}^{\varepsilon} \Vdash 1-\varepsilon \leqslant u$, and so $\left\{v_{u}^{\varepsilon}: u \in A_{\varepsilon}\right\}$ is a partition of unity that witnesses the fact that the net $\left\{a_{E}\right\}$ is $\mathscr{B}$-Cauchy. The same argument shows that $a=\mathscr{B}$-lim $a_{E}$ and so $a \in \mathscr{A}_{0}$.

We now turn our attention to complete subalgebras:

8.12 Definition. $\mathscr{A}_{1}$ is a complete subalgebra of a complete Stonean algebra $\mathscr{A}_{2}$ if $\mathscr{A}_{1}$ is a complete Stonean algebra, with the same operations (including infinite suprema) as $\mathscr{A}_{2}$.

8.13 LemMA. (a) Let $\mathscr{A}_{1}$ be a complete subalgebra of $\mathscr{A}_{2}$ and let $\mathscr{B}_{1}$ and $\mathscr{B}_{2}$ be, respectively, the Boolean algebras of projections in $\mathscr{A}_{1}$ and in $\mathscr{A}_{2}$. Then $\mathscr{B}_{1}$ is a complete Boolean subalgebra of $\mathscr{B}_{2}$.

(b) The reals in $\mathscr{A}_{1}$ are all the $\int \lambda d p_{\lambda}$, where $\left\{p_{\lambda}: \lambda \in \mathbf{R}\right\} \subset \mathscr{B}_{1}$.

(c) Let $\left\{a_{n}\right\}$ be a net in $\mathscr{A}_{1}$. Then $\left\{a_{n}\right\}$ is $\mathscr{B}$-convergent in $\mathscr{A}_{1}$ if and only if it $\mathscr{B}$-convergent as a net in $\mathscr{A}_{2}$ (and has the same $\mathscr{B}$-limit).

Proof. (a) and (b) follow from earlier consideration.

(c) One direction is trivial: if $\left\{a_{n}\right\}$ is $\mathscr{B}$-convergent in $\mathscr{A}_{1}$, then it is $\mathscr{B}$-convergent in $\mathscr{A}_{2}$. The other direction is a consequence of absoluteness of the property " $\left\{a_{n}\right\}$ is a Cauchy net of complex numbers" applied to the Boolean-valued models $V^{\mathscr{B}_{2}}$ and its inner model $V^{\mathscr{B}_{1}}$.

8.14 TheOREM. Let $G$ be a subset of a complete Stonean algebra $\mathscr{A}$. Then there is a smallest complete subalgebra $\mathscr{A}_{0}$ of $\mathscr{A}$ that contains $G$. Moreover:

(a) Let $\mathscr{B}$ be the Boolean algebra of projections in $\mathscr{A}$. The Boolean algebra $\mathscr{B}_{0}$ of projections in $\mathscr{A}_{0}$ is the complete subalgebra of $\mathscr{B}$ generated by the projections $p_{\lambda}$ in spectral resolutionds $\int \lambda d p_{\lambda}$ of (real and imaginary parts of ) elements of $G$.

(b) $\mathscr{A}_{0}$ is the closure of $G$ under the finite operations,$+ \cdot$, scalar multiplication, *, $\wedge$ and $\vee$, as well as $\mathscr{B}$-limits of nets.

(c) If $\mathscr{A}$ is $\sigma$-saturated, then "nets" in (b) can be replaced by "sequences".

8.15 Definition. $\mathscr{A}_{0}$ is the complete subalgebra of $\mathscr{A}$ generated by $G$.

Proof. If $a$ is real and $a=\int \lambda d p_{\lambda}$, then $p_{\lambda}=\llbracket a \leqslant \lambda \rrbracket$, computed in any complete subalgebra of $\mathscr{A}$ that contains $a$. Using this fact, (a) follows easily.

To prove (b) and (c), let $\mathscr{A}_{0}$ be the complete subalgebra generated by $G$, and let $A$ be the closure of $G$ under the finite algebraic operations and $\mathscr{B}$-limits. Clearly, $A \subseteq \mathscr{A}_{0}$ (here we use 8.13(c)).

First, we prove that $\llbracket a>0 \rrbracket \in A$ for every real $a \in A$. Let $b=(a \vee 0) \wedge 1$, and $c=1-b$. Then $0 \leqslant c \leqslant 1$ and $\llbracket a>0 \rrbracket=\llbracket c<1 \rrbracket$. We use the fact that

$$
\llbracket c=1 \rrbracket=\lim _{n \rightarrow \infty} c^{n}
$$

and hence $\llbracket a>0 \rrbracket \in A$.

It follows that $\llbracket a \leqslant \lambda \rrbracket \in A$ whenever $a$ is the real (imaginary) part of some $g \in G$. These are generators of the complete Boolean subalgebra $\mathscr{B}_{0}$ of $\mathscr{B}$. The elements of $\mathscr{B}_{0}$ can be obtained from the generators by successive applications of finite Boolean operations and suprema of sets of mutually orthogonal elements. The latter operation can be replaced by $\mathscr{B}$-limits of nets; moreover, if $\mathscr{B}$ is $\sigma$-saturated, 
then nets can be replaced by sequences. Thus $A$ contains $\mathscr{B}_{0}$. Now every real in $\mathscr{A}_{0}$ has the form

$$
a=\int \lambda d p_{\lambda}=\sup _{\lambda \in \mathbf{R}} \lambda \cdot p_{\lambda}=\sup _{\lambda \in \mathbf{Q}} \lambda p_{\lambda}
$$

( $Q$ is the set of all rational numbers), where $p_{\lambda} \in \mathscr{B}_{0}$. It follows that $a$ is the $\mathscr{B}$-limit of a net in $A$.

\section{Measurable Stonean algebras.}

9.1 Definition. A Stonean algebra $\mathscr{A}$ is measurable if it admits a measure, that is, a $\sigma$-additive probabilistic measure $\mu$ on the set of all $u \geqslant 0$ such that:

(i) $\mu(0)=0, \mu(1)=1$,

(ii) $\mu(u)>0$ for all $u>0$,

(iii) if $u \leqslant v$, then $\mu(u) \leqslant \mu(v)$, and

(iv) if $u_{i} u_{j}=0$ for all distinct $i, j \in I$, then

$$
\mu\left(\bigvee_{i \in I} u_{i}\right)=\sum_{i \in I} u_{i}
$$

The following lemma is immediate.

9.2 LEMMA. The following are equivalent:

(a) $\mathscr{A}$ is measurable.

(b) $\overline{\mathscr{A}}$, the completion of $\mathscr{A}$, is measurable.

(c) $\mathscr{B}$, the Boolean algebra of projections of $\overline{\mathscr{A}}$, is a measure algebra.

(For definition of measure algebra, see [9].)

The function $\mu$ from 9.1 extends to a strictly positive $\sigma$-additive probabilistic measure on $\mathscr{B}$.

9.3 Lemma. Every measurable Stonean algebra is $\sigma$-saturated.

Proof. Every measure algebra has the countable chain condition [9].

This allows to restrict questions related to convergence to sequences (instead of nets).

9.4 THEOREM F. Every measurable complete Stonean algebra is isomorphic to the algebra of all measurable functions $(\bmod =$ a.e.) on some measure space $\mathscr{X}$; bounded elements correspond to functions in $\mathscr{L}_{\infty}(\mathscr{X})$.

Proof. This theorem, for Boolean-valued complex numbers given by measure algebras, is essentially due to Scott and Solovay; cf. [11].

Let $\mathscr{A}$ be a measurable complete Stonean algebra, let $\mathscr{B}$ be its algebra of projections, and let $\mu$ be a measure on $\mathscr{B}$. Let $\mathscr{X}$ be the Stone space of $\mathscr{B}$, and denote also by $\mu$ the complete measure induced by $\mu$ (and $M$ the $\mu$-measurable subsets of $\mathscr{X}) . \mathscr{X}=(\mathscr{X}, \mu, M)$ is a measurable space [3].

Let $\mathscr{M}(\mathscr{X})$ be the set of all complex-valued functions defined almost everywhere on $\mathscr{X}$. Following [11], we associate with each real-valued $f \in \mathscr{M}(\mathscr{X})$ the (unique) $\mathscr{B}$-valued real $a_{f}$ such that

$$
\llbracket a_{f} \leqslant \lambda \rrbracket=[\{x \in \mathscr{X}: f(x) \leqslant \lambda\}], \quad \lambda \in \mathbf{Q},
$$


where the equivalence class $[S]$ denotes the element of $\mathscr{B}$ when $\mathscr{B}$ is identified with $\mathscr{X} /$ null sets. If $f=g$ a.e. then $a_{f}=a_{g}$.

Every $a \in \mathbf{R}^{\mathscr{B}}$ is represented this way: Let $a=\int \lambda d p_{\lambda}$. There exists $\left\{S_{\lambda}: \lambda \in \mathbf{Q}\right\}$ such that, for each $\lambda \in \mathbf{Q},\left[S_{\lambda}\right]=p_{\lambda}, S_{\lambda}=\bigcap_{\varepsilon>0} S_{\lambda+\varepsilon}$ and $\bigcap_{\lambda \in \mathbf{Q}} S_{\lambda}=\varnothing$. For $x \in \cup_{\lambda \in \mathbf{Q}} S_{\lambda}$, let

$$
f(x)=\sup \left\{\lambda \in \mathbf{Q}: x \notin S_{\lambda}\right\}
$$

then $a=a_{f}$.

The correspondence $f \rightarrow a_{f}$ is an isomorphism (between the real functions in $\mathscr{M}(\mathscr{X})$ and $\left.\mathbf{R}^{\mathscr{D}}\right)$ - see [11, Lemma 4.4] - and induce an isomorphism between $\mathscr{M}(\mathscr{X})$ and $\mathscr{A}$. Also,

$$
[\{x \in \mathscr{X}: f(x)=g(x)\}]=\llbracket a_{f}=a_{g} \rrbracket .
$$

The bounded elements of $\mathscr{A}$ correspond to those $a \in \mathbf{C}^{\mathscr{B}}$ for which there is a $\lambda$ such that $\llbracket|a| \leqslant \lambda \rrbracket=1$. In the representation by measurable functions, $a=a_{f}$ where $|f(x)| \leqslant \lambda$ a.e. Hence bounded elements of $\mathscr{A}$ correspond to essentially bounded functions in $\mathscr{M}(\mathscr{X})$.

9.5 TheOREM. A sequence $\left\{f_{n}\right\}$ in $\mathscr{M}(\mathscr{X}) \mathscr{B}$-converges to $f$ if and only if $f_{n}(x) \rightarrow f(x)$ a.e.

Proof. First assume that $f_{n} \stackrel{\mathscr{B}}{\rightarrow} 0$. For each $k$ there is a partition $W_{k}$ and $\left\{N_{S}^{k}\right.$ : $\left.S \in W_{k}\right\}$ such that, for each $S \in W_{k}$ and every $n \geqslant N_{S}^{k},\left|f_{n}(x)\right|<1 / k$ a.e. on $S$. We may assume that $\left|f_{n}(x)\right|<1 / k$ everywhere on $S$ and that the $S \in W_{k}$ are pairwise disjoint; and $Y_{k}=\bigcup\left\{S: S \in W_{k}\right\}$ has full measure. Let $Y=\bigcup_{k=1}^{\infty} Y_{k}$. The set $Y$ has full measure, and if $x \in Y$, then for each $k$ there is a $S \in W_{k}$ such that $x \in S$, and so $f_{n}(x)$ converges to 0 .

Conversely, assume that $f_{n}(x) \rightarrow 0$ a.e. For every $k$ and $N$, let

$$
\mathbf{A}_{N}^{k}=\left\{x \in \mathscr{X}: f(x) \rightarrow 0 \text { and } N \text { is the least } N \text { such that } \forall n \geqslant N\left|f_{n}(x)\right| \leqslant 1 / k\right\} .
$$

For each $k$, the $A_{N}^{k}$ are disjoint measurable sets. Those of positive measure form the partition $W_{k}$; that attests to $\mathscr{B}$-convergence.

We remark that by Egoroff's Theorem [3], if $f_{n} \rightarrow f$ a.e., then $f_{n} \rightarrow f$ piecewise uniformly. See also Theorem 9.8 below.

9.6 Definition [9]. A complete Boolean algebra $\mathscr{B}$ is weakly $\left(\boldsymbol{\aleph}_{0}, \infty\right)$-distributive if it satisfies the following distributive law:

$$
\bigwedge_{k=1}^{\infty} \bigvee_{i \in I} p_{k i}=\bigvee_{\substack{\left\{F_{k}\right\}_{k-1}^{\infty} \\ F_{k} \subseteq I \text { finite }}} \bigwedge_{k=1}^{\infty} \bigvee_{i \in F_{k}} p_{k i}
$$

We call a complete Stonean algebra weakly distributive if its algebra of projections satisfies (9.6).

9.7 LEMMA [9]. Every measure algebra is weakly $\left(\boldsymbol{\aleph}_{0}, \infty\right)$-distributive.

We remark that $C(0,1)$ is not weakly distributive. 
The import of the following theorem is that for measurable Stonean algebras, the two notions of convergence, $\mathscr{B}$-convergence and piecewise uniform convergence coincide.

9.8 TheOREM. Let $\mathscr{A}$ be a complete Stonean algebra. $\mathscr{A}$ is weakly distributive if and only if every $\mathscr{B}$-convergent net is piecewise uniformly convergent.

Proof. Let us first assume that $\mathscr{A}$ is weakly distributive. Definition 9.6 can be reformulated as follows:

9.9. For every sequence $\left\{W_{k}\right\}_{k=1}^{\infty}$ of partitions of unity and very $p \in \mathscr{B}, p>0$, there is a $q \in \mathscr{B}, 0<q \leqslant p$ and a sequence $\left\{F_{k}\right\}_{k=1}^{\infty}$ of finite sets, $F_{k} \subseteq W_{k}$, such that

$$
q \leqslant \bigvee\left\{r: r \in F_{k}\right\} \quad(k=1,2, \ldots) .
$$

Let $\left\{a_{n}\right\}$ be a $\mathscr{B}$-convergent net. For each $k$, let $W_{k}$ be a partition (for $\varepsilon=1 / k$ ) that witnesses $\mathscr{B}$-convergence and let $\left\{N_{r}^{k}: r \in W_{k}\right\}$ be such that $n \geqslant N_{r}^{k}$ implies $\left|r a_{n}-r a\right| \leqslant 1 / k$.

Let $W$ be a maximal set of pairwise orthogonal projections $q$ such that there exists a sequence $\left\{F_{q}^{k}\right\}_{k=1}^{\infty}$ of finite $F_{k} \subseteq W_{k}$ that satisfy (9.10). By weak distributivity (9.9), $W$ is a partition of unity. We claim that, for each $q \in W,\left\{q a_{n}\right\}$ converges uniformly.

Fix $q \in W$. For each $k$, let

$$
N=\max \left\{N_{r}^{k}: r \in F_{k}\right\} .
$$

By (9.10), if $n \geqslant N$, then $\left|q a_{n}-q a\right| \leqslant 1 / k$, and so $\left\{q a_{n}\right\}$ converges uniformly.

Conversely, let us assume that $\mathscr{A}$ is not weakly distributive and let $p_{0},\left\{W_{k}\right\}_{k=1}^{\infty}$ be a counterexample to 9.9 . It follows that every $q \in \mathscr{B}, 0<q \leqslant p_{0}$, meets infinitely many $r \in W_{k}$ for some $k$.

We assume that each $W_{k}$ is countable and construct a sequence that is $\mathscr{B}$-convergent but not piecewise uniformly convergent. A similar idea works for nets in the general case. We also assume, without loss of generality, that $p_{0}=1$.

Let $W_{k}=\left\{p_{k n}\right\}_{n=1}^{\infty}$. For each $k$ and each $n$, let $a_{k n}=p_{k n} / k$ and let us consider the sequence $\left\{a_{k n}\right\}$. First we show that $\left\{a_{k n}\right\} \mathscr{B}$-converges to 0 : Let $k$ be given, let $W$ be a common refinement of $W_{1}, \ldots, W_{k}$ and let $q \in W$. For each $j \leqslant k, q$ meets a unique $p \in W_{j}$ (in fact $q \leqslant p$ ); say $p=p_{j, n(j)}$. Now if $(i, n)$ is any $(i, n)$ except for finitely many values $(1, n(1)),(2, n(2)), \ldots,(k, n(k))$, then either $i \leqslant k$ and $q a_{i n}=0$ (becausc $n \neq n(i)$ ), or $i>k$ and $q a_{i n} \leqslant 1 / i$. In either case, $q a_{i n} \leqslant 1 / k$. Hence $\mathscr{B}-\lim a_{k n}=0$.

We now show that $\left\{a_{k n}\right\}$ does not converge piecewise uniformly. Let $W$ be any partition of unity and let $q \in W$. There is a $k$ such that $q$ meets infinitely many $p_{k n} \in W_{k}$. Hence $\left\|q a_{k n}\right\|=1 / k$ for infinitely many $n$, and so $\left\{q a_{k n}\right\}$ does not converge uniformly to 0 .

To conclude this section we remark that the results can be generalized to the case when $\mathscr{B}$ is not a measure algebra but a direct sum of measure algebras. $\mathscr{A}$ is not necessarily $\sigma$-saturated and the measure $u$ on $\mathscr{X}$ is not necessarily $\sigma$-finite. 
10. Complete abelian algebras of operators. We shall now apply the general theory to commuting normal operators in a Hilbert space. Let $\mathscr{H}$ be a Hilbert space and let us consider normal, not necessarily bounded, operators on $\mathscr{H}$.

10.1 Definition. A partition of unity is a maximal set of mutually orthogonal projections. A partition of unity $W$ is a support for a normal operator $A$ if, for every $P \in W, A P$ is a bounded operator and $A P=P A P$.

Two normal operators $A$ and $B$ are compatible if they have a common support $W$, and $A P$ commutes with $B P$, for every $P \in W$.

The following theorem lists well-known facts about normal operators:

10.2 TheOREM. (a) Every normal operator has the form $A+i B$, where $A$ and $B$ are compatible selfadjoint operators.

(b) Every selfadjont operator has a (unique) spectral resolution

$$
A=\int \lambda d P_{\lambda}
$$

(c) Two normal operators are compatible if and only if the projections in their respective spectral resolutions mutually commute.

10.3 Lemma. Let $W$ be a partition of unity and let $A_{P}$ be, for each $P \in W$, a normal operator such that $A_{P} P=P A_{P} P$. Then there exists a unique normal $A$ such that $A_{P} P=A P$ for all $P \in W$.

We use the notation

$$
A=\sum_{P \in W} A_{P} P
$$

10.5 Definition. Let $A$ and $B$ be compatible normal operators, with support $W$ :

(a) $A+B=\sum_{P \in W}(A P+B P)$,

(b) $A \cdot B=\sum_{P \in W}(A P \cdot B P)$, and

(c) $A \leqslant B$ if $A P \leqslant B P$ for all $P \in W$.

The definition of,$+ \cdot$ and $\leqslant$ is independent of the choice of common support. The operations + and . have the usual algebraic properties and $\leqslant$ is a partial ordering. Moreover, if $S$ is a nonempty set of mutually compatible selfadjoint operators that has an upper bound (in $\leqslant$ ), then it has a least upper bound, $\sup S$, which is a normal operator compatible with all $A \in S$.

10.6 Definition. A complete abelian algebra of operators $\mathscr{A}$ is a mutually compatible set of normal operators that is closed under $+, \cdot,^{*}$ and scalar multiplication and such that $\sup S \in \mathscr{A}$ for every nonempty $S \subset \mathscr{A}$ that has an upper bound in $\leqslant$.

10.7 Proposition. (a) Every complete abelian algebra of operators is a complete Stonean algebra (with,$+ \cdot$ and $\leqslant$ defined in 10.5 , and the usual * and scalar multiplication).

(b) If $\mathscr{A}$ is a mutually compatible set of normal operators that is (under,$+ \cdot$, etc. and sup) a complete Stonean algebra, then $\mathscr{A}$ is a complete abelian algebra of operators. 
Proof. (a) A routine verification.

(b) The only reason why this is not entirely trivial is that we have to verify that if $S \subseteq \mathscr{A}$ has an upper bound in $\leqslant$, then $S$ has an upper bound in $\mathscr{A}$. Let $A=\sup S$ and let $A=\int \lambda d P_{\lambda}$ be the spectral resolution of $A$. For each $\lambda$,

$$
P_{\lambda}=\bigwedge\{\llbracket X \leqslant \lambda \rrbracket: X \in S\} \in \mathscr{A},
$$

and so $A \in \mathscr{A}$.

10.8 RemarK. If $\mathscr{A}$ is a complete abelian algebra of operators, then $B(\mathscr{A})$ is a von Neumann algebra.

THEOREM E. Given a set $G$ of mutually compatible normal operators there is a smallest complete abelian algebra $\mathscr{A}$ such that $G \subseteq \mathscr{A}$.

$W e$ say that $\mathscr{A}$ is generated by $G$.

Proof. Let $\mathscr{B}$ be the complete Boolean algebra generated (in the lattice of projections) by the set of all projections in spectral resolutions of all operators in $G$. Let $\mathscr{A}(G)$ be the set of all normal operators whose spectral projections are in $\mathscr{B}$. $\mathscr{A}(G)$ is a complete Stonean algebra, and hence a complete abelian algebra of operators. If $\mathscr{A}$ is any complete abelian algebra of operators, then $\mathscr{A}$ is a complete Stonean algebra and, by Theorem $8.14, \mathscr{A}(G)$ is the complete subalgebra of $\mathscr{A}$ generated by $G$; hence $\mathscr{A}(G) \subseteq \mathscr{A}$.

If $\mathscr{A}_{0}$ is an abelian von Neumann algebra of operators in $\mathscr{H}$, then the complete abelian algebra $\mathscr{A}$ generated by $\mathscr{A}_{0}$ consists of all normal operators affiliated with $\mathscr{A}_{0}$ (in the terminology of [5]). Also, $\mathscr{A}$ is the completion of $\mathscr{A}_{0}$, and $\mathscr{A}_{0}=B(\mathscr{A})$. If $G$ consists of a single unbounded normal operator, then $\mathscr{A}$ is isomorphic to the algebra constructed in [2].

10.9. The kind of convergence usually associated with operators is the (strong) operator convergence. It is easy to construct an example of a sequence of normal operators converging to zero which is not $\mathscr{B}$-convergent. As for the converse, we note the following lemma.

10.10 Lemma (TAKeUTI [15]). If $\left\{A_{n}\right\}$ is a $\mathscr{B}$-convergent net of compatible normal operators with limit $A$ and if there is a $\lambda$ such that $\left\|A_{n}-A\right\| \leqslant \lambda$ for all $n$, then $A_{n}$ converges strongly to $A$ (i.e. $A_{n}-A \rightarrow 0$ ).

Proof. Assume that $\left\{A_{n}\right\} \stackrel{\mathscr{B}}{\rightarrow} 0$. Let $x \in \mathscr{H}$ and $\varepsilon>0$. There is a partition of unity $W$ and $\left\{N_{P}: P \in W\right\}$ such that for all $P \in W$ and $n \geqslant N P,\left\|A_{n}-P\right\|$ is small relative to $\|x\|$. Let $F \subseteq W$ be finite such that $\sum_{P \in W-F} A_{n} P$ is small. Then let $N=\max \left\{N_{P}: P \in F\right\}$, etc.

10.11. Let $G$ be a mutually commuting set of normal bounded operators and let $\mathscr{A}$ be the complete abelian algebra generated by $G$. By Theorem $8.14, \mathscr{A}$ is the closure of $G$ under the finite algebraic operations and $\mathscr{B}$-limits of nets. The bounded operators in $\mathscr{A}$ can be obtained as $\mathscr{B}$-limits of nets bounded by some $\lambda$, and so it follows by Lemma 10.10 that $B(\mathscr{A})$ is the closure of $G$ under $+, \cdot, *, \vee, \wedge$, scalar multiplication and strong limits of nets. The unbounded operators in $\mathscr{A}$ are then obtained from $\mathscr{B}(\mathscr{A})$ as $\sum_{P \in W} A_{P} P$ where the $P$ 's and $A_{p}$ 's belong to the von Neumann algebra $\mathscr{B}(\mathscr{A})$. 
As will be discussed shortly, every complete abelian algebra of operators is weakly distributive, and so every $\mathscr{B}$-convergent net is piecewise uniformly convergent. It follows that if $\mathscr{A}$ is generated by $G$, then $\mathscr{A}$ is the closure of $G$ under the finite algebraic operations and piecewise uniform limits of nets.

10.12. The structure of any complete Stonean algebra $\mathscr{A}$ is determined by its Boolean algebra $\mathscr{B}$ of projections. In the case when $\mathscr{A}$ is an algebra of operators, $\mathscr{B}$ is not arbitrary. The Stone space $\mathscr{X}$ of $\mathscr{B}$ admits $\sigma$-additive Borel measures and it follows that $\mathscr{B}$ is the direct sum of (possibly uncountably many) measure algebras ( $\mathscr{X}$ has an open dense set which is a disjoint union of $\sigma$-finite measure spaces; $\mathscr{X}$ is hyperstonean [14]). One consequence is that $\mathscr{B}$ is weakly distributive. Measure algebras have been classified in [7]: every measure algebra is a direct sum of homogeneous measure algebras, and every measure algebra is essentially given by the product measure on the measure space $\{0,1\}^{\kappa}$ for some $\kappa$. This provides a complete classification of complete abelian algebras of operators.

10.13. In the particular case when $\mathscr{A}$ is a complete abelian algebra of operators on a separable Hilbert space, and if $\mathscr{B}$ is atomless, then $\mathscr{B}$ is a countably generated atomless measure algebra, which is unique up to isomorphism, and so is isomorphic to the algebra of Borel sets of reals mod Lebesgue measure zero sets. Then $\mathscr{A}$ is isomorphic to the algebra of all measurable functions on $\mathbf{R}(\bmod =$ a.e. $)$ and $B(\mathscr{A})$ corresponds to $\mathscr{L}^{\infty}(\mathbf{R})$.

10.14 Definition. A complete Stonean algebra $\mathscr{A}$ has a complete representation on a Hilbert space $\mathscr{H}$ if $\mathscr{A}$ is isomorphic to a complete abelian algebra of operators in $\mathscr{H}$.

The preceding discussion reveals that a necessary condition for $\mathscr{A}$ to have a complete representation on a Hilbert space is that $\mathscr{A}$ is the direct sum of measurable aglebras. Thus, for instance, the completion of $C[0,1]$ does not have a complete representation because its Boolean algebra (Borel sets of reals mod meager sets) is not weakly distributive.

THEOREM G. Every measurable complete Stonean algebra $\mathscr{A}$ has a complete representation on some Hilbert space $\mathscr{H}$; if $\mathscr{A}$ is countably generated, then there is an $\mathscr{H}$ that is separable.

Proof. The construction is, of course, well known. Let $\mathscr{B}$ be the Boolean algebras of projections, let $\mathscr{X}$ be the Stone space of $\mathscr{B}$, and let $\mathscr{H}=\mathscr{L}_{2}(\mathscr{X}) . \mathscr{A}$ is isomorphic to $\mathscr{M}(\mathscr{X})$, and every $f \in \mathscr{M}(\mathscr{X})$ is represented by the multiplication operator $A_{f}(g)=$ $f g$.

Added in proof (November 1984). 1. Professor Manasao Ozawa of the Tokyo Institute of Technology has kindly brought to my attention existing work related to the subject of this paper. In particular, the theory of vector lattices, or Riesz spaces (cf. W. A. J. Luxemburg and A. C. Zaanen, Riesz spaces, North-Holland, Amsterdam, 1971) is very close to our approach. In view of the representation of complete Stonean algebras by normal functions (Theorem B), and a theorem of F. Maeda and T. Ogasawara (cf. Representation of vector lattices, J. Sci. Hiroshima Univ. (A) 12 
(1942), 17-35), the real part of a complete Stonean algebra is a universally complete Dedekind complete Archimedean Riesz space (in the terminology of Luxemburg and Zaanen).

2. It was also pointed out to me by Professor Ozawa that in view of the Representation Theorem B, the bounded part of a complete Stonean algebra is exactly what is generally known as commutative AW* algebra (cf. I. Kaplansky, Algebras of type I, Ann. of Math. 56 (1952), 460-472). I wish to add that in Boolean valued analysis and type I AW* algebras, Proc. Japan Acad. Ser. A 59 (1983), 368-371, Ozawa gives the first application of forcing in this area. Ozawa's result underscores the role of Boolean valued models.

3. $B$-convergence is weaker than the order convergence, which is the convergence usually associated with Riesz spaces (they coincide however for monotone sequences). For instance, the analog of Theorem D fails for order convergence.

\section{REFERENCES}

1. P. J. Cohen, The independence of the continuum hypothesis, Proc. Nat. Acad. Sci. U.S.A. 50 (1963), $1143-1148$.

2. J. M. G. Fell and J. L. Kelley, An algebra of unbounded operators, Proc. Nat. Acad. Sci. U.S.A. 38 (1952), 592-598.

3. P. R. Halmos, Measure theory, Springer-Verlag, New York, 1974.

4. T. Jech, Set theory, Academic Press, New York, 1978.

5. R. V. Kadison and J. R. Ringrose, Fundamentals of the theory of operator algebras, Vol. I, Academic Press, New York, 1983.

6. J. L. Kelley, General topologv, Van Nostrand, Princeton, N. J., 1955.

7. D. Maharam, On homogeneous measure algebras, Proc. Nat. Acad. Sci. U.S.A. 28 (1942), 108-111.

8. F. Riesz and B. Sz.-Nagy, Functional analysis, Ungar, New York, 1955.

9. R. Sikorski, Boolean algebras, Springer-Verlag, 1964.

10. R. M. Solovay, A model of set theory in which every set of reals in Lebesgue measurable, Ann. of Math. (2) 92 (1970), 1-56.

11. Real-valued measurable cardinals, Axiomatic Set Theory (D. Scott, ed.), Proc. Sympos. Pure Math., Vol. 13, part 1, Amer. Math. Soc., Providence, R. I., 1971, pp. 397-428.

12. M. H. Stone, The theory of representations for Boolean algebras, Trans. Amer. Math. Soc. 40 (1936), $37-111$.

13. A general theory of spectra, I, Proc. Nat. Acad. Sci. U.S.A. 26 (1940), 280-283.

14. M. Takesaki, Theory of operator algebras. I, Springer-Verlag, New York, 1979.

15. G. Takeuti, Two applications of logic to mathematics, Princeton Univ. Press, Princeton, N. J., 1978.

16. P. Vopěnka, The limits of sheaves over extremally disconnected compact Hausdorff spaces, Bull. Acad. Polon. Sci. Sér. Sci. Math. 15 (1967), 1-4.

Department of Mathematics, Pennsylvania State University, University Park, Pennsylvania 16802 